\title{
ALGEBRAIC RESULTS ON REPRESENTATIONS OF SEMISIMPLE LIE GROUPS
}

\section{J. LEPOWSKY $\left.{ }^{1}\right)$}

ABSTRACT. Let $G$ be a noncompact connected real semisimple Lie group with finite center, and let $K$ be a maximal compact subgroup of $G$. Let 8 and l denote the respective complexified Lie algebras. Then every irreducible representation $\pi$ of $g$ which is semisimple under $\mathbb{f}$ and whose irreducible $P$ components integrate to finite-dimensional irreducible representations of $K$ is shown to be equivalent to a subquotient of a representation of 8 belonging to the infinitesimal nonunitary principal series. It follows that $\pi$ integrates to a continuous irreducible Hilbert space representation of $G$, and the best possible estimate for the multiplicity of any finite-dimensional irreducible representation of $P$ in $\pi$ is determined. These results generalize similar results of Harish-Chandra, R. Godement and J. Dixmier. The representations of $g$ in the infinitesimal nonunitary principal series, as well as certain more general representations of 8 on which the center of the universal enveloping algebra of 8 acts as scalars, are shown to have (finite) composition series. A general module-theoretic result is used to prove that the distribution character of an admissible Hilbert space representation of $G$ determines the existence and equivalence class of an infinitesimal composition series for the representation, generalizing a theorem of N. Wallach. The composition series of Weylgroup-related members of the infinitesimal nonunitary principal series are shown to be equivalent. An expression is given for the infinitesimal spherical functions associated with the nonunitary principal series. In several instances, the proofs of the above results and related results yield simplifications as well as generalizations of certain results of Harish-Chandra.

1. Introduction. In his famous "subquotient theorem" [6(c), p. 63, Theorem 4], Harish-Chandra showed that a certain large class of irreducible representations of a semisimple Lie algebra can be realized as subquotients (i.e., quotients of subrepresentations) of certain standard representations. The purpose of this paper is to sharpen and simplify this theorem, and to study related questions. We shall work with a noncompact connected real semisimple Lie group $G$ with finite center (although many of our results could be extended to the case of infinite center), and we shall use algebraic techniques to investigate

Received by the editors December 18, 1971 and, in revised form, May 10, 1972. AMS (MOS) subject classifications (1970). Primary 22E45, 17B10, 20G05, 16 A64.

Key words and phrases. Real semisimple Lie group, real semisimple Lie algebra, irreducible representation, subquotient, nonunitary principal series, multiplicity, composition series, distribution character, Weyl group, spherical function, universal enveloping algebra, admissible Hilbert space representation, infinitesimal equivalence, maximal compact subgroup, Iwasawa decomposition.

( $\left.{ }^{1}\right)$ This paper was written while the author was partially supported by NSF GP 28323. 
the representations of $G$ and of its Lie algebra. Several of our results had been obtained by Harish-Chandra, R. Godement and J. Dixmier in the special case in which $G$ is a linear group. The treatment here is based on the study of various maps related to a certain map $P$ (see below)-essentially the map $\xi$ introduced by Harish-Chandra in his proof of the subquotient theorem [6(c), p. 48]. This study leads us to generalizations, and sometimes simplifications, of HarishChandra's analysis of a map $\gamma$ (see [6(g), p. 260]) used extensively by him and other authors to study spherical representations of $G$. After the work for this paper had been completed, we learned that C. Rader [13] had independently obtained results very close to some of our main results, including a similar generalization of Harish-Chandra's subquotient theorem, using some of the same methods. Our treatment and Rader's are different in many respects, however.

In order to describe our main results, we introduce some notation. Let $K$ be a maximal compact subgroup of $G$, and let $€ \subset g$ denote the respective complexified Lie algebras. Let $\oint_{\text {b }}$ be the universal enveloping algebra of g. A (complex) $\biguplus$-module $V$ which is also a $K$-module is called a $(乌, K)$-module if

$$
k \cdot(x \cdot v)=(k \cdot x) \cdot(k \cdot v)
$$

for all $k \in K, x \in Y_{\text {and }} v \in V$. (Module actions are denoted with a dot, and $K$

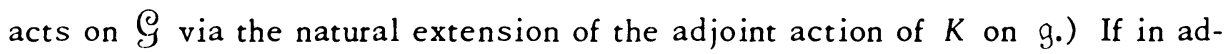
dition $K \cdot v$ spans a finite-dimensional space on which $K$ acts differentiably for all $v \in V$, and the action of $\notin \subset \oint$ on any finite-dimensional $K$-invariant subspace of $V$ is the action induced by the differential of the action of $K$, then $V$ is said to be compatible.

Let $G=K A N$ be an Iwasawa decomposition of $G$ adapted to $K$, and let $M$ be the centralizer of $A$ in $K$. The nonunitary principal series of $G$ is the family of continuous Hilbert space representations of $G$ induced in the standard way (see $\$ 7$ ) from the (not necessarily unitary) finite-dimensional irreducible representations of the subgroup $M A N$ of $G$. If $\pi$ is a continuous Banach space representation of $G$ whose restriction to $K$ contains every irreducible representation of $K$ with at most finite multiplicity, the corresponding space of vectors whose $K$-transforms span a finite-dimensional space (that is, the space of $K$ -

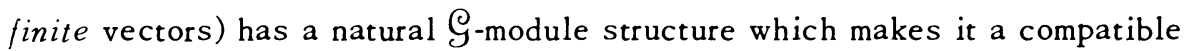
(乌, K)-module-the infinitesimal representation associated with $\pi$. Two such Banach space representations of $G$ are said to be infinitesimally equivalent if their infinitesimal representations are equivalent.

Our main result is the following:

Theorem 1.1 (see Theorem 8.10). Every 乌-irreducible compatible (乌, K)module is equivalent to the $(乌, K)$-module of $K$-finite vectors of some continuous 
topologically irreducible (not necessarily unitary) representation of $G$ on a Hilbert space, and this representation may be chosen to be a subquotient (that is, a quotient of subrepresentations on closed subspaces) of a nonunitary principal series representation. Every continuous topologically irreducible Banach space representation of $G$ which contains every irreducible representation of $K$ with at most finite multiplicity is infinitesimally equivalent to an irreducible subquotient of a nonunitary principal series representation.

This theorem is basically a generalization of Harish-Chandra's subquotient theorem [6(c) p. 63, Theorem 4] from the case of linear $G$ to the case of arbitrary $G$ (with finite center), in the following sense: Harish-Chandra's theorem is stated for $g$ and not $G$, but it contains a hypothesis (namely, that "some $\mathscr{I}_{0} \epsilon$ $\Omega_{F}$ occurs in $\pi^{\prime \prime}$ ) which in effect restricts it to a class of groups $G$ intermediate between the linear groups and the arbitrary ones. But Harish-Chandra uses a different definition of the nonunitary principal series from ours, and his proof implies Theorem 1.1 only for linear groups $G$. The reason is that his proof depends on Lemma 1, p. 28 of [6(c)], which is essentially a result about linear groups, in view of its hypothesis concerning finite-dimensional representations of g. Our proof of Theorem 1.1 is essentially a simplification of Harish-Chandra's proof, but there is one major change-Lemma 1 of $[6(c)]$ is replaced by a result (The orem 1.3(b) below) applicable to arbitrary $G$. This enables us to remove the linearity assumption on $G$. (Theorem 1.3(b) also allows us to avoid using Theorem 1, p. 195 of [6(b)].) Using more difficult analytic techniques, HarishChandra has also proved the last assertion of Theorem 1.1 for arbitrary $G$ (see [6(h), Theorem 4]).

The following result is a corollary of Theorem 1.1, but in fact it can be proved directly, much more easily:

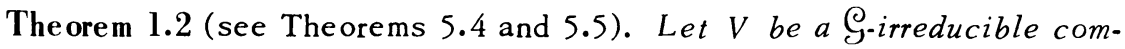
patible (乌, K)-module, and let $\beta$ be an equivalence class of finite-dimensional irreducible representations of $K$. Then the multiplicity with which members of $\beta$ occur in $V$ is equal to or less than the maximum of the multiplicities with which irreducible representations of $M$ occur in any member of $\beta$, and this estimate is the best possible. This bound is equal to or less than the degree of any member of $\beta$, and is finite. The same result bolds when $V$ is replaced by a continuous topologically irreducible Banach space representation of $G$ which contains every irreducible representation of $K$ with at most finite multiplicity.

The last assertion of Theorem 1.2 had been proved by Harish-Chandra since it follows from the last assertion of Theorem 1.1. Moreover, the first assertion 
had been proved by Harish-Chandra for a class of groups intermediate between the linear groups and the arbitrary ones, in view of his subquotient theorem (see the discussion following Theorem 1.1). But as we have indicated, our proof is much simpler and more direct. It is based on Theorem 1.3(b) below and the "minimal standard identity" technique used by R. Godement $[5, \S 1]$ and J. Dixmier ([3(a)] and [3(b), §15.5]) to prove a result similar to the last assertion of The orem 1.2 for linear groups $G$. Harish-Chandra had obtained a weaker estimate [6(c), p. 36, Theorem 3] for the $K$-multiplicities associated with $V$, in the notation of the first part of The orem 1.2.

Let $\mathcal{G}^{K}$ denote the centralizer of $K$ in $\mathcal{G}$, let $\mathcal{K}$ be the universal envelop-

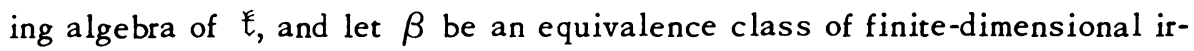
reducible representations of $K$. Any member of $\beta$ naturally induces a representation of $\mathcal{K}$, and we denote its kernel by $g^{\beta}$, so that $g^{\beta}$ is a maximal two-sided ideal of $\mathcal{K}$. Then $\mathcal{G}^{K} \cap \mathscr{G}^{\beta}$ is a two-sided ideal of the algebra $\mathcal{G}^{K}$, and the algebra $\mathcal{G}^{K} / \mathcal{G}^{K} \cap \mathcal{G}^{\beta}$ has considerable significance, since its irreducible representations correspond exactly to the irreducible representations of $\varrho$ whose restriction to $\mathcal{K}$ contains members of $\beta$ with positive multiplicity, in view of [11, Theorem 5.5]. We do not know the full structure of the algebra $\varrho^{K} / \varrho^{K} \cap$ gg $\beta$, but we shall say something about it.

Let $a$ and $n$ denote the complexified Lie algebras of $A$ and $N$, and let $\mathscr{A}$ and $\pi$ denote the universal enveloping algebras of $a$ and $\pi$. Then there is a natural linear isomorphism of $\mathcal{G}$ with $\Re \otimes \mathbb{Q} \otimes \mathcal{K}$, and it follows that we may write

$$
\mathcal{S}=\mathfrak{A} \otimes \mathcal{K} \oplus n \mathcal{H}
$$

Let

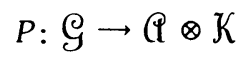

be the corresponding projection map, and regard $\mathscr{Q} \otimes \mathcal{K}$ as an algebra in the natural way. Let $\pi_{\beta}: \mathcal{K} \rightarrow \mathcal{K} / g^{\beta}$ denote the quotient map, and let

$$
P_{\beta}=\left(1 \otimes \pi_{\beta}\right) \circ P: \mathcal{G} \rightarrow \mathbb{Q} \otimes K / g^{\beta} .
$$

Also, let $K^{M}$ denote the centralizer of $M$ in $\mathcal{K}$ with respect to the adjoint action. Then we have

Theorem 1.3 (see Propositions 3.1, 3.2 and 3.3, and Corollaries 4.4 and 4.5). (a) The restriction of $P$ to $\mathcal{G}^{K}$ is an algebra antibomomorpbism which injects $\mathrm{G}^{K}$. into $\mathbb{Q} \otimes \mathcal{K}^{\mathrm{M}}$.

(b) The restriction of $P_{\beta}$ to $\mathcal{G}^{K}$ is an algebra antibomomorphism with kermel precisely $\mathcal{G}^{K} \cap \mathcal{G}^{\beta}=\mathcal{G}^{K} \cap \mathscr{G}^{\beta} \mathcal{G}$, and $P_{\beta}$ takes $\mathcal{G}^{K}$ into $\left(\oplus \otimes\left(\mathcal{K}^{M} / \mathcal{K}^{M} \cap \mathscr{g}^{\beta}\right)\right.$. 
In particular, $P_{\beta}$ induces an injection

$$
\mathrm{S}^{K} / \mathcal{G}^{K} \cap \mathrm{gg}^{\beta} \hookrightarrow \mathbb{Q} \otimes\left(\mathcal{K}^{M} / \mathcal{K}^{M} \cap \mathrm{g}^{\beta}\right)
$$

which is an algebra antibomomorphism.

The last assertion of Theorem 1.3(b) implies that $\mathcal{G}^{K} / \mathcal{G}^{K} \cap \mathcal{G g}^{\beta}$ satisfies a certain polynomial identity, and this easily implies Theorem 1.2.

Suppose that $\beta$ is the class of the trivial one-dimensional representation of $K$. Then $P_{\beta}: \mathcal{G}^{K} \rightarrow \mathbb{Q}$ is essentially the same as the mapping $\gamma$ studied by Harish-Chandra in $[6(\mathrm{~g}), \S 4]$. Our proof of the equality $\mathcal{G}^{K} \cap \mathcal{G g}^{\beta}=\mathcal{G}^{K} \cap g^{\beta} \mathcal{G}$ is easier than Harish-Chandra's proof of the corresponding equality $\varrho^{K} \cap \varrho^{E}=$

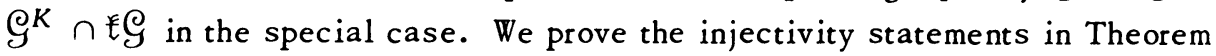
1.3 by generalizing the simplified proof of Harish-Chandra's special case given in [7(a), p. 431, Lemma 6.13]. (We also give an alternate proof of the injectivity statement in (a).)

Define the linear map $p_{\beta}: \mathcal{G} \rightarrow \mathbb{A}$ by $p_{\beta}=\left(1 \otimes \operatorname{tr}_{\beta}\right) \circ P_{\beta}$, where $\operatorname{tr}_{\beta}$ denotes the canonical trace map on the full matrix algebra $\mathcal{K} / \mathscr{g} \beta$. In Theorem 10.1, we compute the image of the map $p_{\beta}: \mathcal{G}^{K} \rightarrow \mathbb{Q}$, generalizing Harish-Chandra's corresponding results $[6(\mathrm{~g})$, p. 260, Theorem 1 and p. 262, Lemma 19] for the map $\gamma$. The image of $p_{\beta}$ is essentially the set of Weyl group invariants in $\mathbb{P}$.

We also define other mappings associated with $P$, and we relate them to the "infinitesimal spherical functions" associated with the representations in the nonunitary principal series and with the finite-dimensional irreducible representations of $G$ (see Theorems 6.5 and 7.3 and Remark 6.6), extending related results of Harish-Chandra (cf. [6(c), p. 49, Lemma 11], [9, Lemma 1.2.7] and [12, Theorem 2.2 (2.34) and Theorem 2.3 (2.55)]). Our proofs are based on an extension of the notion of highest weight vector (see $\$ 6$ ); this idea was inspired by B. Kostant (see [9, Lemmas 1.7.5 and 1.7.6]).

We show that every representation in the infinitesimal nonunitary principal series has a (finite) composition series (Theorem 9.7); the hard part of the proof is due to Harish-Chandra, in the form of his deep theorem [6(i)] that distribution characters are locally summable functions. (Several people have suggested using Harish-Chandra's results to prove Theorem 9.7.) Theorem 9.7 is a consequence of a stronger result (Theorem 9.16) relating various finiteness condi-

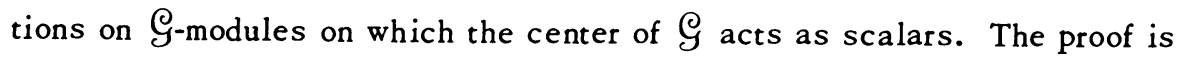
based on Theorem 1.1 as well as Harish-Chandra's local summability theorem. Part of The orem 9.16 was obtained by Harish-Chandra and Dixmier for linear groups $G$ [3(c), Lemme 1 and Lemme 2].

Using Harish-Chandra's character formula for the representations in the 
nonunitary principal series, we show that Weyl-group-related members of the infinitesimal nonunitary principal series have equivalent composition series, and we relate this fact to spherical functions, infinitesimal spherical functions and certain generalizations of highest weight vectors (Theorem 9.8). This equivalence of composition series is a special case of a general principle (Theorem 9.3) relating distribution characters of admissible Hilbert space representations to the existence and equivalence of infinitesimal composition series. The proof of this theorem (which generalizes a result of $\mathrm{N}$. Wallach) is based in turn on a much more general module-theoretic result (Theorem 11.1), which is the subject of the Appendix.

As an alternative to using Harish-Chandra's character formula to prove the equivalence of composition series indicated above, we outline a proof based on the formal intertwining integrals of R. A. Kunze and E. M. Stein for the nonunitary principal series, F. Bruhat's irreducibility result for the regular members of the unitary principal series and an algebraic continuity argument (see Remark 9.11). When this idea is applied to the spherical nonunitary principal series, it simplifies to a new, rather short, proof of the fact that the image of HarishChandra's map $\gamma$ (see above) lies in the set of Weyl group invariants in ( 9 (see Remark 9.12).

At the end of $\S 10$, we state two conjectures concerning the images of $P_{\beta}$ and related maps.

We are very grateful to Bertram Kostant for suggesting the problem of generalizing Harish-Chandra's map $\gamma$, providing some helpful ideas concerning the generalization, informing us of Dixmier's Lemma 2.2, and allowing us to read his manuscript of [9].

2. Preliminaries on modules. The notation and terminology introduced in this section will be used throughout the paper without explicit reference.

Algebras will be associative algebras with identity element 1 , and representations of groups, algebras or Lie algebras will be left representations. The representation space $V$ of a representation of a group, algebra or Lie algebra $A$ will be called a module over $A$, and the action will often be indicated by $a \cdot v(a \in A, v \in V)$. Modules over real Lie groups, complex algebras and complex Lie algebras are assumed to be complex vector spaces. The equivalence class of a module $V$ is denoted by $[V]$. Let $\mathbf{C}$ be the field of complex numbers. The dual of a complex vector space $V$ is written as $V^{\prime}$, and the pairing between $V$ and $V^{\prime}$ is denoted by $\langle\cdot, \cdot\rangle$. The restriction of a function $f$ to a subset $X$ of its domain will be written as $f \mid X$.

Let $A$ be a real Lie group and let $V$ be an $A$-module. An element $v \in V$ is called $A$-finite if $A \cdot v$ spans a finite-dimensional space on which the action 
of $A$ is differentiable. $V$ is called $A$-finite if every element of $V$ is $A$-finite. Let $\hat{A}$ denote the set of equivalence classes of differentiable finite-dimensional irreducible $A$-modules. For every $\alpha \in \hat{A}$, let $V_{\alpha}$ denote the $\alpha$-primary subspace of the $A$-module $V$, that is, the subspace of the space of $A$-finite vectors in $V$ consisting of the sum of all the irreducible $A$-submodules of $V$ in the class $\alpha$. Let $m([V], \alpha)$ denote the multiplicity with which members of a occur in $V$.

Suppose that $A$ is a Lie subgroup of a real Lie group $B$. Let $b$ be the complexified Lie algebra of $B$, and let $\mathscr{B}$ be the universal enveloping algebra of $\mathfrak{b}$. We identify $\mathfrak{b}$-modules and $\mathfrak{B}$-modules in the usual way. $B$ acts in a canonical way as a group of algebra automorphisms of $\mathcal{B}$, by unique extension of the adjoint representation of $B$ on its Lie algebra. In particular, $A$ acts on $B$ as a group of algebra automorphisms, by restriction. For every subset $S$ of $B$ and every subset $\mathcal{T}$ of $\mathfrak{B}$, let $\mathcal{T}^{S}$ denote the centralizer of $S$ in $\mathfrak{T}$. We regard $B$-modules as $A$-modules by restriction, so that for every $\beta \in \hat{B}$ and $\alpha \in \hat{A}$, $m(\beta, \alpha)$ is the multiplicity with which members of $\alpha$ occur in any member of $\beta$.

Let $A_{1}$ be a subgroup of $A$ and let $B_{1}$ be an $A_{1}$-invariant subalgebra of $B$. Let $V$ be a $\Re_{1}$-module and an $A_{1}$-module. We call $V$ a $\left(\mathscr{B}_{1}, A_{1}\right)$-module if

$$
a \cdot(b \cdot v)=(a \cdot b) \cdot(a \cdot v)
$$

for all $a \in A_{1}, b \in \Re_{1}$ and $v \in V$. ( $\left.\Re_{1}, A_{1}\right)$-module maps and equivalence of $\left(B_{1}, A_{1}\right)$-modules are defined in the obvious ways. The equivalence class of a $\left(B_{1}, A_{1}\right)$-module $V$ is denoted by $[V]$.

Let $V$ be a $(\mathscr{B}, A)$-module, let $\alpha \in \hat{A}$ and let $X$ be a fixed module in the class $a$. Then the action of $A$ on $V$ commutes with the action of $B^{A}$, so that the space $\operatorname{Hom}_{A}(X, V)$ is a $\mathfrak{B}^{A}$-module in a natural way, by the action of $\mathfrak{B}^{A}$ on $V$. We denote the corresponding equivalence class of $\mathfrak{B}^{A}$-modules by $\eta([V], \alpha)$.

Lemma 2.1. Suppose that $A$ is compact and that $V$ is a B-irreducible (,$A)$-module. Let $\alpha \in \hat{A}$ and suppose that $V_{\alpha} \neq 0$. Then $\eta([V], \alpha)$ is an irreducible class of $B^{A}$-modules. Furthermore, let $W$ be a finite-dimensional $A$ submodule of $V$, and let $C(W) \subset$ End $W$ denote the commuting ring of the action of $A$ on $W$. Then for all $\mu \in C(W)$, there exists $x \in B^{A}$ such that $x \cdot w=\mu(w)$ for all $w \in W$.

To prove the lemma, we use the following argument due to J. Dixmier:

Lemma 2.2 (Dixmier). Let $S$ be an irreducible set of operators on a vector space $V$ of countable dimension over $\mathrm{C}$. Then the commuting ring of $S$ on $V$ consists of the scalars. In particular, the conclusion bolds if $S$ is an algebra of countable dimension over $\mathrm{C}$ and $V$ is an irreducible S-module. 
Proof. Let $C$ be the commuting ring of $S$ on $V$, and let $v \in V, v \neq 0$. Since $C$ is a division ring, the map $C \rightarrow V$ given by $c \mapsto c \cdot v(c \in C)$ is an injection. Hence $\operatorname{dim} C$ is countable. Moreover, $C$ is a division algebra over C. But we assert that every field over $\mathbf{C}$ not equal to $\mathbf{C}$ has uncountable dimension over $\mathrm{C}$. Indeed, let $F$ be such a field, and let $X \in F$ be transcendental over $\mathrm{C}$. Then $\{1 /(X-\lambda) \mid \lambda \in \mathbf{C}\}$ is an uncountable linearly independent set, proving the assertion. Thus if $x \in C$, then $\mathrm{C}(x)=\mathrm{C}$, so that $x \in \mathrm{C}$. Q.E.D.

Proof of Lemma 2.1. Let $W$ and $\mu$ be as stated. Since $\operatorname{dim} B$ is countable, Lemma 2.2 applies. Hence by the Jacobson density theorem, there exists $u \in \mathcal{B}$ such that $u \cdot w=\mu(w)$ for all $w \in W$. Thus

$$
\mu(a \cdot w)=a \cdot \mu(w)=a \cdot(u \cdot w)=(a \cdot u) \cdot(a \cdot w)
$$

for all $a \in A$ and $w \in W$, so that $\mu(w)=(a \cdot u) \cdot w$ for all $a \in A$ and $w \in W$. Integrating over the compact group $A$, we get $\mu(w)=x \cdot w$, where $x \in \mathfrak{B}^{A}$, proving the last statement.

Let $X \in \alpha$. To show that $\operatorname{Hom}_{A}(X, V)$ is $\mathfrak{B}^{A}$-irreducible, we shall show that if $f, g \in \operatorname{Hom}_{A}(X, V), f \neq 0$, then there exists $x \in \mathfrak{B}^{A}$ such that $x \cdot f=g$. Let $W=f(X)+g(X)$, so that $W$ is a finite-dimensional $A$-submodule of $V$. By the above, it is sufficient to show that there exists $\mu \in C(W)$ such that $\mu \circ f=g$. But if $f(X)=g(X)$, we may choose $\mu$ to be multiplication by a suitable constant on $W$. Finally, if $f(X) \neq g(X)$, then $f(X) \cap g(X)=0$, and we may choose $\mu$ so that $\mu \mid f(X)$ is a suitable $A$-module map from $f(X)$ to $g(X)$ and so that $\mu \mid g(X)=0$. Q.E.D.

Every $\beta \in \hat{B}$ gives rise in a natural way to an equivalence class of ( $(A, A)$ modules, so that for all $\alpha \in \hat{A}, \eta(\beta, \alpha)$ is a well-defined equivalence class of finite-dimensional $\mathfrak{B}^{A}$-modules. If $B$ is connected, then the corresponding class of $(\mathfrak{B}, A)$-modules is $\mathscr{B}$-irreducible, and if $A$ is compact, then by Lemma 2.1, $\eta(\beta, \alpha)$ is either the zero class or an irreducible class.

Let $\mathcal{C}$ be a complex algebra, and let $\alpha$ be an equivalence class of $\mathcal{C}$ modules. We denote by $\operatorname{Ker} a$ the kernel in $\mathcal{C}$ of any representation in the class $\alpha$. If $\alpha$ is a class of finite-dimensional representations of $\mathcal{C}$, then the character $\chi(\alpha): \mathcal{C} \rightarrow \mathrm{C}$ is the linear function defined by $\chi(\alpha)(c)=\operatorname{tr} \pi(c)$ for all $c \in \mathcal{C}$, where $\pi$ is a representation in the class $a$.

Denote by $\mathbb{A}$ the universal enveloping algebra of the complexified Lie algebra of $A$, regarded as embedded in $\mathscr{B}$ in the natural way. $A(B, A)$-module $V$ is called compatible if it is $A$-finite and if the action of $\mathbb{Q}$ on any finitedimensional $A$-invariant subspace of $V$ is the action induced by the differential of the action of $A$.

Let $\alpha \in \hat{A}$, and let $\alpha_{1}$ be the equivalence class of finite-dimensional $\mathcal{A}$ modules induced by $a$. Then we define $\operatorname{Ker} \alpha=\operatorname{Ker} \alpha_{1} \subset \mathbb{A}$. Let $V$ be a 
$(\mathfrak{B}, A)$-module, and let $X \in \alpha$. If $V$ is compatible, then $\mathfrak{B}^{A} \cap \mathfrak{B} \operatorname{Ker} \alpha$ annihilates the $\mathfrak{B}^{A}$-module $\operatorname{Hom}_{A}(X, V)$, so that the equivalence class $\eta([V], \alpha)$ of $\mathfrak{B}^{A}$ modules may be identified with an equivalence class of $\mathfrak{B}^{A} / \mathfrak{B}^{A} \cap \mathfrak{B} \operatorname{Ker} \alpha$ modules. (Note that $\mathfrak{B}^{A} \cap \mathfrak{B} \operatorname{Ker} \alpha$ is a two-sided ideal of $\mathfrak{B}^{A}$.)

Let $b \mapsto b^{t}(b \in \Re)$ denote the transpose map, that is, the unique (involutive) antiautomorphism of $\mathfrak{B}$ which extends -1 on $\mathfrak{b}$. For every $\mathfrak{B}$-module $V$, the contragredient $\mathfrak{B}$-module structure on the dual $V^{\prime}$ is defined by $\left\langle v, b \cdot v^{\prime}\right\rangle=$ $\left\langle b^{t} \cdot v, v^{\prime}\right\rangle$ for all $v \in V, v^{\prime} \in V^{\prime}$ and $b \in \mathfrak{B}$. The restriction to $\mathbb{A}$ of the transpose map of $\mathfrak{B}$ is the transpose map of $\mathcal{A}$. For every equivalence class $a$ of finite-dimensional $\uparrow$-modules, we let $\alpha$ ! denote the contragredient class. Then $\operatorname{Ker} \alpha^{\prime}=(\operatorname{Ker} \alpha)^{t}$.

Let $\hat{\mathbb{Q}}$ denote the set of equivalence classes of finite-dimensional irreducible $\mathfrak{Q}$-modules. For every $\mathbb{Q}$-module $V$ and $\alpha \in \hat{\mathbb{A}}$, we denote by $V_{a}$ the $a$ primary subspace of $V$. An $\mathbb{Q}$-module $V$ is called finitely semisimple if

$$
V=\coprod_{a \in \hat{\mathbb{a}}} V_{\alpha} .
$$

We regard $\mathfrak{B}$-modules as $\mathscr{T}$-modules by restriction.

Lemma 2.3. Let $V$ be a finitely semisimple $\mathfrak{Q}$-module, and let $a \in \hat{\mathbb{Q}}$. If $v \in V_{a}$ and $v \neq 0$, then $\left\langle v,\left(V^{\prime}\right)_{\alpha^{\prime}}\right\rangle \neq 0$.

Proof. Write

$$
V_{\alpha}=\coprod_{i \in I} x_{i}
$$

a direct sum of $\mathbb{P}_{\text {-submodules }} X_{i}$ in the class $\alpha$. For each $i \in I$, we embed $X_{i}^{\prime}$ into $V^{\prime}$ by defining $\left\langle v, x_{i}^{\prime}\right\rangle=0$ for all $x_{i}^{\prime} \in X_{i}^{\prime}$ and

$$
v \in \coprod_{j \in I ; j \neq i} X_{i}+Y,
$$

where $Y$ is the sum of the irreducible $\mathbb{P}$-submodules of $V$ not in the class $\alpha$. This embedding is clearly an $\mathbb{Q}$-module map, and the lemma is now clear. Q.E.D.

We now recall some of the notions of [11]. Let $\alpha \in \hat{\mathbb{U}}$. We define $g^{\alpha}=$ Ker $a \subset \mathbb{P}$, and $A^{\alpha}=\left\{x \in \mathcal{B} \mid g^{a}{ }_{x} \subset B^{\alpha}\right\}$ (cf. [11, 33$]$ ). Then by [11, Propositions 3.2 and 3.3], $\mathcal{B g}^{\alpha}$ (resp. $A^{\alpha}$ ) is precisely the subset of $\mathfrak{B}$ which annihilates (resp., preserves) $V_{a}$ for every $B$-module $V$.

Suppose that $\mathfrak{b}=a \oplus c$ where $a$ is the complexified Lie algebra of the Lie group $A$, and $c$ is an $a$-invariant complement of $a$ in $b$ such that the natural representation of $a$ on $c$ is semisimple. Then for all $a \in \hat{\mathbb{A}}, \mathfrak{B} / \mathfrak{B g}^{\alpha}$ is a finite-

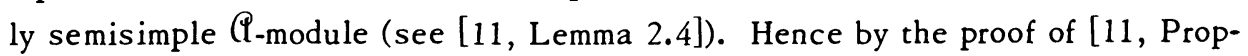
osition 3.2], it follows that $\mathfrak{B g}^{\alpha}$ is precisely the subset of $\mathfrak{B}$ which annihilates $V_{\alpha}$ for every $\mathfrak{B}$-module $V$ which is finitely semisimple under $\mathfrak{Q}$. 
Lemma 2.4. For all $a \in \hat{\mathbb{A}}, A^{a} \cap g^{a} \mathfrak{B} \subset \mathfrak{B g}^{\alpha}$.

Proof. Let $V$ be a $\mathfrak{B}$-module which is finitely semisimple under $\mathbb{A}$, and let $x \in A^{\alpha} \cap g^{\alpha} \mathcal{B}$. Then $x^{t} \in\left(g^{\alpha} \beta\right)^{t}=\mathscr{B}\left(g^{\alpha}\right)^{t}=\mathscr{B} g^{\alpha^{\prime}}$ by the above, so that

$$
\left\langle x \cdot V_{\alpha^{\prime}}\left(V^{\prime}\right)_{\alpha^{\prime}}\right\rangle=\left\langle V_{\alpha^{\prime}} x^{t} \cdot\left(V^{\prime}\right)_{\alpha^{\prime}}\right\rangle=0 .
$$

But $x \in A^{a}$, so that $x \cdot V_{\alpha} \subset V_{\alpha}$. Hence $x \cdot V_{\alpha}=0$ by Lemma 2.3 , so that $x \in$ $B g^{\alpha}$. Q.E.D.

Lemma 2.5.(2) Let $\alpha \in \hat{\mathbb{P}}$, and let $S$ be a transpose-invariant subset of $A^{\alpha} \cap$

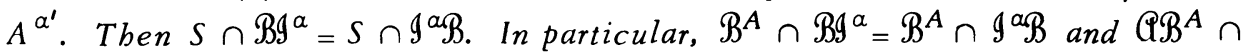
$\mathfrak{B g}^{\alpha}=\mathbb{P B}^{A} \cap \mathfrak{g}^{\alpha} \mathfrak{B}$.

Proof. By Lemma 2.4, $S \cap \mathcal{G}^{\alpha} \mathcal{B} \subset S \cap \mathfrak{B g}^{\alpha}$ and $S \cap \mathfrak{g}^{\alpha^{\prime}} \mathfrak{B} \subset S \cap \mathfrak{B g}^{\alpha^{\prime}}$. The second inclusion gives $\left(S \cap g^{a^{\prime}} \Re\right)^{t} \subset\left(S \cap B^{a^{\prime}}\right)^{t}$, that is, $S \cap B^{a} \subset S \cap g^{a} \beta$. Q.E.D.

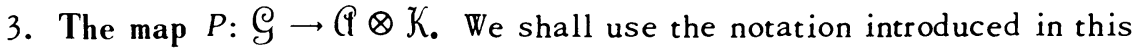
section throughout the paper, often without explicit reference.

Let $G$ be a noncompact connected real semisimple Lie group with finite center, and let $g_{R}$ be its Lie algebra. Fix a Cartan decomposition $g_{R}=E_{R}+$ $p_{R}$ and an associated Iwasawa decomposition $g_{R}=\mathfrak{E}_{R}+a_{R}+n_{R}$ of $g_{R}$, and let $g=\mathfrak{E}+\not$ and $g=\mathfrak{E}+a+n$ be the corresponding complexified decompositions. Let $G=K A N$ be the corresponding Iwasawa decomposition of $G$, so that $K$ is a maximal compact subgroup of $G$. Let $M$ be the centralizer of $A$ in $K$.

Let $\mathcal{G}, \mathcal{K}, \mathbb{Q}$ and $\Re$ be the universal enveloping algebras of $g, \mathcal{E}, a$ and $n$,

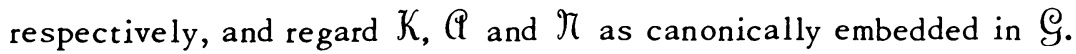

Suppose that $\mathcal{T}_{1}, \mathcal{T}_{2}, \ldots, \mathcal{T}_{j}$ are the universal enveloping algebras of subalgebras $t_{1}, \ldots, t_{j}$ of $g$ such that $g$ is the direct sum of the $t_{i}$, and regard $\mathfrak{T}_{1}, \ldots, \mathfrak{T}_{j}$ as canonically embedded in $\mathcal{S}_{\text {. The the map from }} \mathfrak{T}_{1} \otimes \ldots \otimes \mathfrak{T}_{j}$ to $\mathcal{G}$ given by

$$
t_{1} \otimes \cdots \otimes t_{j} \mapsto t_{1} \cdots t_{j}
$$

is a linear isomorphism. This holds in particular when the $t_{i}$ are $E, a$ and $n$, taken in any order.

We now describe a basic decomposition of $\varrho$ and several mappings associated with it.

We have

$$
\mathcal{S}=\Re \mathbb{P K}=(\mathbf{C} \cdot 1 \oplus n \pi) \mathbb{P K}=\mathbb{A K} \oplus n \mathcal{G} .
$$

Let $P: \mathcal{G} \rightarrow$ IK denote the corresponding projection map.

We give $\mathbb{A K}$ an algebra structure by identifying it with the algebra $\mathbb{P} \otimes \mathcal{K}$,

(2) Lemma 2.5 will be used in the proof of Corollary 4.5. 
and we regard $P$ as a map $P: \subseteq \rightarrow \mathbb{Q} \otimes \mathcal{K}$ (cf. the map $\xi$ introduced by HarishChandra in [6(c), p. 48]).

Proposition 3.1 (cf. [6(c), p. 48, Lemma 10]). For all $u \in \mathcal{Y}$ and $v \in \oint^{K}$, we bave $P(u v)=P(v) P(u)$. In particular, the restriction $P \mid \biguplus^{K}$ is an algebra antibomomorphism.

Proof. Write

$$
\begin{aligned}
& u \equiv \sum_{i} a_{i} x_{i} \quad\left(\bmod n Y_{)},\right. \\
& v \equiv \sum_{j} b_{j} y_{j} \quad\left(\bmod n Y_{)},\right.
\end{aligned}
$$

where $a_{i}, b_{j} \in \mathbb{P}$ and $x_{i}, y_{j} \in \mathcal{K}$. Then

(since $v \in \varrho^{K}$ )

$$
u v \equiv \sum_{i} a_{i} v x_{i} \quad\left(\bmod n \varrho_{)}\right.
$$

$$
\equiv \sum_{i, j} a_{i} b_{j} y_{j} x_{i} \quad\left(\bmod n Y_{)}\right)
$$

(since a normalizes $n$ )

$$
\equiv P(v) P(u) \quad(\bmod n \biguplus) . \quad \text { Q.E.D. }
$$

Proposition 3.2. $P: \mathscr{G}^{M} \rightarrow \mathbb{A} \otimes \mathcal{K}^{M}$, and in particular, $P: \mathscr{G}^{K} \rightarrow \mathbb{Q} \otimes \mathcal{K}^{M}$.

Proof. This follows immediately from the fact that $M$ normalizes $n$ and centralizes a. Q.E.D.

Proposition 3.3. $P: \mathscr{G}^{K} \rightarrow \mathbb{C} \otimes \mathcal{K}^{M}$ is an injection, or equivalently, $\mathcal{G}^{K} \cap$ $n \mathcal{Y}=0$.

Proof. It is sufficient to show that $\mathcal{G}^{K} \cap n \mathcal{G}=0$. To prove this, let $V$ be any finite-dimensional irreducible $g$-module. Let $v^{\prime} \in V^{\prime}$ be a highest restricted weight vector of the contragredient $g$-module $V^{\prime}$, that is, a nonzero element $v^{\prime}$ $\epsilon V^{\prime}$ such that $n \cdot v^{\prime}=0$ and such that $x \cdot v^{\prime}$ is a scalar multiple of $v^{\prime}$ for all $x \in a$. Let $u \in \mathcal{G}^{K} \cap n \mathcal{~}$. Then

$$
\begin{aligned}
0 & =\left\langle V, u^{t} \cdot v^{\prime}\right\rangle=\left\langle u \mathcal{K} \cdot V, v^{\prime}\right\rangle=\left\langle\mathcal{K} u \cdot V, v^{\prime}\right\rangle \\
& =\left\langle u \cdot V, \mathcal{K} \cdot v^{\prime}\right\rangle=\left\langle u \cdot V,\left(\mathcal{K} \mathscr{Q}+\complement_{n}\right) \cdot v^{\prime}\right\rangle=\left\langle u \cdot V, \mathfrak{S} \cdot v^{\prime}\right\rangle=\left\langle u \cdot V, V^{\prime}\right\rangle,
\end{aligned}
$$

so that $u \cdot V=0$. Thus $u=0$ since $\biguplus$ has "sufficiently many" representations [6(a), Theorem 1]. Q.E.D.

Hence the map $P$ "antiembeds" $\mathcal{G}^{K}$ as a subalgebra of $\mathbb{Q} \otimes K^{M}$. A different proof of Proposition 3.3 will be given in $\$ 4$. 
Fix $\beta \in \hat{K}$, and let $g^{\beta}=\operatorname{Ker} \beta$, so that $g^{\beta}$ is a maximal two-sided ideal of $\mathcal{K}$. Let $\pi_{\beta}: \mathcal{K} \rightarrow \mathcal{K} / \mathcal{g}^{\beta}$ be the projection map. Define $P_{\beta}: \mathcal{G} \rightarrow \mathbb{A} \otimes \mathcal{K} / g^{\beta}$ by $P_{\beta}=\left(1 \otimes \pi_{\beta}\right) \circ P$. Identifying $\pi_{\beta}\left(\mathcal{K}^{M}\right)$ with $\mathcal{K}^{M} / \mathcal{K}^{M} \cap g^{\beta}$, we have $P_{\beta}: \mathcal{G}^{M}$ $\rightarrow \mathbb{Q} \otimes\left(\mathcal{K}^{M} / \mathcal{K}^{M} \cap g^{\beta}\right)$, and in particular, $P_{\beta}: \mathcal{G}^{K} \rightarrow \mathbb{Q} \otimes\left(\mathcal{K}^{M} / \mathcal{K}^{M} \cap g^{\beta}\right)$. Furthermore, $P_{\beta}$ is an antihomomorphism on $\mathcal{G}^{K}$.

Let $\operatorname{tr}_{\beta}: \mathcal{K} / g^{\beta} \rightarrow \mathrm{C}$ denote the canonical trace map on the algebra $K / g^{\beta}$, which is isomorphic to a full matrix algebra over $\mathrm{C}$. Define the linear map

$$
p_{\beta}=\left(1 \otimes \operatorname{tr}_{\beta}\right) \circ P_{\beta}: \mathcal{Y} \rightarrow \mathbb{Q} \text {. }
$$

Let $\Gamma_{\beta}=\{\gamma \in \hat{M} \mid m(\beta, \gamma)>0\}$, and for all $\gamma \in \Gamma_{\beta}$, let $g^{\beta, \gamma}=\operatorname{Ker} \eta(\beta, \gamma)$ in $\mathcal{K}^{M}$. Then $\mathcal{G}^{\beta, \gamma}$ is a maximal two-sided ideal of $\mathcal{K}^{M}$ since $\eta(\beta, \gamma)$ is an irreducible class (see $\$ 2$ ).

Now $K^{M} / \mathcal{K}^{M} \cap g^{\beta}$ is isomorphic to the commuting ring of the action of $M$ on any $K$-module in the class $\beta$, and so $\mathcal{K}^{M} / \mathcal{K}^{M} \cap g^{\beta}$ is isomorphic to the direct product of the matrix algebras $K^{M} / \mathcal{I}^{\beta}, \gamma\left(\gamma \in \Gamma_{\beta}\right)$. More precisely, it is clear that $\mathcal{K}^{M} \cap g^{\beta}=\bigcap_{\gamma \in \Gamma_{\beta}} g^{\beta, \gamma}$. Let $f_{\beta}: K^{M} \rightarrow \Pi_{\gamma \in \Gamma_{\beta}} K^{M} / \mathcal{I}^{\beta, \gamma}$ be the homomorphism induced by the projections $\pi_{\beta, \gamma}: K^{M} \rightarrow K^{M} / \mathcal{G}^{\beta, \gamma}$. Then

$$
\operatorname{Ker} f_{\beta}=\bigcap_{\gamma \in \Gamma_{\beta}} g^{\beta, \gamma}=K^{M} \cap g^{\beta},
$$

and so $f_{\beta}$ induces an isomorphism $K^{M} / K^{M} \cap g^{\beta} \frown \Pi_{\gamma \in \Gamma_{\beta}} K^{M} / \mathcal{I}^{\beta, \gamma}$. Hence we may regard

$$
P_{\beta}: \mathscr{G}^{M} \rightarrow @ \otimes \prod_{\gamma \in \Gamma_{\beta}} K^{M} / \mathcal{I}^{\beta, \gamma},
$$

or

$$
P_{\beta}: \mathfrak{S}^{M} \rightarrow \prod_{\gamma \in \Gamma_{\beta}} \oplus \otimes \mathcal{K}^{M} / \mathcal{I}^{\beta, \gamma}
$$

Thus

$$
P_{\beta} \mid \varrho^{M}=\prod_{\gamma \in \Gamma_{\beta}} P_{\beta, \gamma},
$$

where, for fixed $\gamma \in \Gamma_{\beta}, P_{\beta, \gamma}: \mathcal{S}^{M} \rightarrow \mathbb{Q} \otimes K^{M} / \mathcal{I}^{\beta, \gamma}$ is defined by $P_{\beta, \gamma}=$ $\left(1 \otimes \pi_{\beta, \gamma}\right) \circ P$. Then $P_{\beta, \gamma} \mid \mathcal{G}^{K}$ is an ant ihomomorphism.

Now let $\operatorname{tr} \beta, \gamma: K^{M} / g^{\beta, \gamma} \rightarrow \mathbf{C}$ denote the canonical trace map on the full matrix algebra $K^{M} / \mathcal{I}^{\beta, \gamma}$. Define the linear map

$$
P_{\beta, \gamma}=\left(1 \otimes \operatorname{tr}_{\beta, \gamma}\right) \circ P_{\beta, \gamma}: \mathcal{G}^{M} \rightarrow \mathbb{A}
$$


For every $\gamma \in \hat{M}$, let $d(\gamma)$ denote the dimension of any module in $\gamma$. For every subset $\Gamma \subset \Gamma_{\beta}$, define

$$
p_{\beta, \Gamma}=\sum_{\gamma \in \Gamma} d(\gamma) p_{\beta, \gamma}: \varrho^{M} \rightarrow \mathbb{A} .
$$

Then $p_{\beta} \mid \mathcal{G}^{M}=p_{\beta, \Gamma_{\beta}}$, since every module in $\beta$, when regarded as a $\mathcal{K}^{M}$. module, is the direct sum, over $\gamma \in \Gamma_{\beta}$, of $d(\gamma)$ copies of a module in $\eta(\beta, \gamma)$.

For all $\lambda \in a^{\prime}$, let $\pi_{\lambda}: \mathbb{P} \rightarrow \mathbb{C}$ be the evaluation homomorphism at $\lambda$, where $\mathbb{A}$ is identified with the algebra of polynomial functions on $a^{\prime}$. We define

$$
\begin{aligned}
& P_{\lambda}=\left(\pi_{\cdot \lambda} \otimes 1\right) \circ P: \mathcal{G} \rightarrow \mathcal{K}, \\
& P_{\beta, \lambda}=\left(\pi_{\lambda} \otimes 1\right) \circ P_{\beta}: \varrho \rightarrow K / g^{\beta} \text {, } \\
& p_{\beta, \lambda}=\pi_{\lambda} \circ p_{\beta}: 乌 \rightarrow C, \\
& P_{\beta, \gamma, \lambda}=\left(\pi_{\lambda} \otimes 1\right) \circ P_{\beta, \gamma}: \mathcal{G}^{M} \rightarrow K^{M} / \mathcal{I}^{\beta, \gamma}, \\
& p_{\beta, \gamma, \boldsymbol{\lambda}}=\pi_{\lambda} \circ p_{\beta, \gamma}: \mathcal{S}^{M} \rightarrow \mathbf{C} \text {, } \\
& p_{\beta, \Gamma, \lambda}=\pi_{\lambda} \circ p_{\beta, \Gamma}: \varrho^{M} \rightarrow \mathbf{C} \text {. }
\end{aligned}
$$

Then $P_{\lambda}\left|\varrho^{K}, P_{\beta, \lambda}\right| \varrho^{K}$ and $P_{\beta, \gamma, \lambda} \mid \varrho^{K}$ are antihomomorphisms.

Remark 3.4. Let $\beta$ be the class of the trivial one-dimensional representation of $K$. Then $g^{\beta}=\mathcal{K}$ and $P_{\beta}=p_{\beta}$ is the classical map which takes $x \in \mathcal{G}$ to the $\mathbb{P}$-component of $x$ with respect to the decomposition $\biguplus=\mathbb{Q} \oplus\left(\varrho^{E}+n \biguplus\right)$ (cf. [G(g), p. 247, Lemma 3], [7(a), p. 427, Lemma 6.6] and [9, Proposition 1.2.2]).

Remark 3.5. The antihomomorphisms $P_{\beta, \gamma}: \mathcal{G}^{K} \rightarrow \mathbb{Q} \otimes \mathcal{K}^{M} / \mathcal{G}^{\beta, \gamma}$ may be regarded as generalizations of the maps $h^{Q}$ in $[12, \S 2.3]$.

4. The kernel of the map $P_{\mathfrak{g}} \mid \mathrm{G}^{K}$. Let 9 be an arbitrary two-sided ideal of $\mathcal{K}$, and let $\pi_{g}: \mathcal{K} \rightarrow \mathcal{K} / \mathcal{G}$ denote the projection map. Define the linear map $P_{\mathfrak{g}}: \mathcal{G} \rightarrow \mathbb{Q} \otimes K / \mathcal{K}$ by $P_{\mathfrak{g}}=\left(1 \otimes \pi_{\mathfrak{g}}\right) \circ P$, so that $P_{\mathfrak{g}} \mid \mathcal{G}^{K}$ is an algebra antihomomorphism, by Proposition 3.1. In this section, we shall compute the kernel of $P_{\mathfrak{g}} \mid \mathcal{G}^{K}$, and in later sections, we shall apply the result to representation theory.

For any vector space $V$, let $S(V)$ denote the symmetric algebra over $V$. Let $\lambda: S(g) \rightarrow \varrho$ denote the "symmetrization" mapping, that is, the unique linear isomorphism such that

$$
\lambda\left(x_{1} \cdots x_{n}\right)=\frac{1}{n !} \sum_{\sigma} x_{\sigma(1)} \cdots x_{\sigma(n)}
$$

for all nonnegative integers $n$ and all $x_{1}, \ldots, x_{n} \in g$ (see $[1, \S 2.7]$ or $[6(b)$, p. 192]). Here $\sigma$ ranges over all permutations of $\{1, \ldots, n\}$, the product on 
the left is taken in $S(g)$, and the products on the right are taken in $\mathcal{G}$. We note that $\lambda$ is defined on $S(p)$ by regarding $S(p) \subset S(g)$. Similarly, $\lambda$ is defined on $S(a)$, and in fact the map $\lambda: S(a) \rightarrow \mathbb{A}$ is an algebra isomorphism since $a$ is abelian.

Now $S(g)$ and $\varrho$ are both $G$-modules, by unique extension by automorphisms of the adjoint representation of $G$ on $g$, and it is clear that $\lambda$ is a $G$-module isomorphism. In particular, $S(p)$ is a $K$-submodule of $S(g), \lambda(S(p))$ is a $K$-submodule of $\mathcal{G}$, and $\lambda \mid S(\not)$ is a $K$-module isomorphism of $S(\beta)$ onto $\lambda(S(\xi))$.

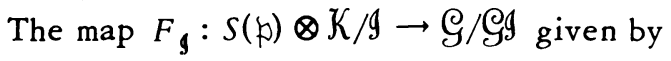

$$
x \otimes(y+9) \mapsto \lambda(x) y+\varrho g
$$

$(x \in S(p), y \in \mathcal{K})$ is a linear isomorphism (see [11, Lemma 2.3] or [6(b), pp. 195$196])$. For every nonnegative integer $i$, let $S^{i}(p)$ denote the $i$ th degree homogeneous subspace of $S(p)$, so that

$$
\text { G/g }=\coprod_{i=0}^{\infty} F_{g}\left(S^{i}(\not) \otimes K \mathcal{K} / g\right) .
$$

For all nonzero $x \in \mathscr{G} / \mathcal{G}$, define $\operatorname{deg} x$ to be the smallest nonnegative integer $n$ such that

$$
x \in \coprod_{i=0}^{n} F_{9}\left(S^{i}(p) \otimes K / g\right)
$$

also define $\operatorname{deg} 0=-\infty$.

Let $q_{R}$ be the orthogonal complement of $a_{R}$ in $p_{R}$ with respect to the Killing form of $g_{R}$, and let $q \subset p$ be the complexification of $q_{R}$. Then $S(p)=$ $S(a) \oplus q S(p)$, so that

$$
\mathcal{G} / \mathcal{G}=F_{g}(S(a) \otimes K / g) \oplus F_{g}(q S(\not) \otimes K / g) .
$$

Let $f_{\mathrm{g}}$ denote the projection of $\mathcal{G} / \mathcal{G}$ onto the first summand in this decomposition.

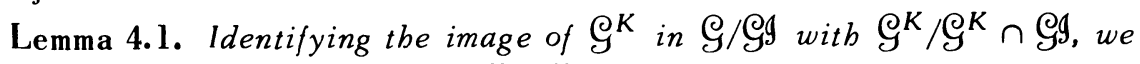
bave that the restriction of $f_{g}$ to $\varrho^{K} / \mathcal{G}^{K} \cap \varrho^{g}$ is injective. Moreover, $\operatorname{deg} f_{g}(x)=\operatorname{deg} x$ for all $x \in \varrho^{K} / \mathcal{G}^{K} \cap 乌_{\text {. }}$

Proof. Let $g: S(k) \rightarrow S(a)$ be the projection with respect to the decomposition $S(p)=S(a) \oplus q S(p)$, and let $b_{g}=g \otimes 1: S(p) \otimes K / g \rightarrow S(a) \otimes K / g$.

Regard $\mathcal{K}$ as a $K$-module by unique extension by automorphisms of the adjoint representation of $K$ on $E$, and give $S(\xi) \otimes K / g$ a $K$-module structure by regarding it as the tensor product of the $K$-module $S(\xi)$ with the quotient $K$ module $K / \mathscr{G}$. Then it is clear that $F_{g}$ is a $K$-module isomorphism, where $\mathcal{G} / \mathcal{G}$ is regarded as a $K$-module by means of the quotient of the natural representation 
of $K$ on $\mathscr{G}$. Moreover, since $\mathcal{G}$ is a semisimple $K$-module, $\mathcal{G}^{K} / \mathcal{G}^{K} \cap \mathrm{G}^{g}$ is precisely the set of $K$-invariants in $\mathcal{G} / \mathcal{G}$. Let $I$ denote the set of $K$-invariants in $S(\xi) \otimes K / 9$, so that $F_{g}: I \rightarrow \varrho^{K} / \varrho^{K} \cap \varrho_{g}$ is an isomorphism. It is clear that to prove the first statement, it is sufficient to show that $b_{g} \mid I$ is injective.

Now $S(\not)$ may be identified with the algebra of polynomial functions on $k^{\prime}$. Furthermore, the restriction to $p$ of the Killing form of $g$ is nonsingular, and thus may be used to identify $p$ with $k^{\prime}$. Hence we may identify $S(\beta)$ with the algebra of polynomial functions on $\wp$. In the same way, we may identify $S(a)$ with the algebra of polynomial functions on $a$. Under these identifications, $g$ is simply the restriction map to $a$. Moreover, the action of $K$ on $S(\not)$ is identified with the usual action of $K$ on the space of polynomial functions on $p$, since the Killing form of $g$ is $K$-invariant.

Choose a basis $\left\{x_{i}\right\}$ of $\mathcal{K} / \mathscr{I}$, and let

$$
y=\sum_{i} w_{i} \otimes x_{i} \in I
$$

$\left(w_{i} \in S(p)\right)$ be such that $b_{g}(y)=0$, that is, $\Sigma_{i} w_{i}(u) x_{i}=0$ for all $u \in a$. Let $v \in$ $p_{\mathrm{R}}$. Since $K \cdot a_{\mathrm{R}}=\mathfrak{p}_{\mathrm{R}}($ see $[7(\mathrm{a}), \mathrm{p} .211$, Lemma 6.3]), there exists $k \in K$ and $u \in a_{\mathrm{R}}$ such that $k \cdot v=u$. Now $k \cdot y=y$, so that

$$
\sum_{i} w_{i}\left(k^{-1} \cdot u\right) k \cdot x_{i}=0 \text {. }
$$

But since $\left\{k \cdot x_{i}\right\}$ is a basis of $\mathcal{K} / 9$, we have $w_{i}(v)=0$, so that $w_{i}\left(\mathfrak{p}_{\mathrm{R}}\right)=0$, for each $i$. Since the $w_{i}$ are polynomial functions, this implies that $w_{i}=0$, and so $y=0$, proving the first statement.

The second statement now follows immediately from the fact that the direct sum

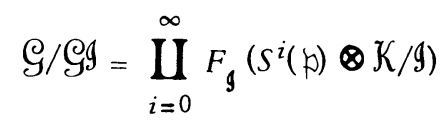

is a $K$-module decomposition, so that

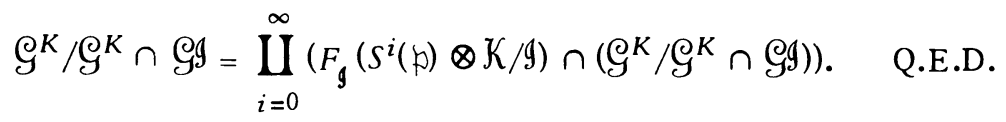

We note that $\mathcal{G}_{\mathfrak{g}} \subset \operatorname{Ker} P_{\mathfrak{g}}$, since

$$
\mathscr{G} 9=r \mathscr{A K} g=r \mathscr{A} \subset \mathbb{P} 9+n \mathscr{G} \text {. }
$$

Regard $P_{g}$ as a map on $\mathcal{G}_{/} / \mathcal{G}$, and let

$$
P_{\mathfrak{g}}^{*}=F_{\mathfrak{g}} \circ\left(\lambda^{-1} \otimes 1\right) \circ P_{\mathfrak{g}}: \mathscr{G}_{/} \mathscr{G}_{g} \rightarrow F_{\mathfrak{g}}(S(a) \otimes K / g) .
$$


Lemma 4.2. For all $x \in \mathcal{G} / \mathcal{G}, \operatorname{deg}\left(P_{g}^{*}(x)-f_{g}(x)\right)<\operatorname{deg} x$.

Proof. Since $P_{g}^{*}$ is the identity on $F_{g}(S(a) \otimes K / g)$, it follows from the definition of $f_{g}$ that it is sufficient to show that, for all $y \in F_{g}(q S(p) \otimes K / g)$, we have $\operatorname{deg} P_{g}^{*}(y)<\operatorname{deg} y$. Let $d=\operatorname{deg} y$, and for every nonnegative integer $a$, let $S_{a}(g)$ denote the sum of the homogeneous subspaces of $S(g)$ of degree $\leq a$. Since $q C$ $E^{k} n$ (see [7(a), p. 223, Lemma 3.6]), we have

$$
y \in\left(\lambda\left(S_{d-1}(\not) \mathcal{E}\right) \mathcal{K}+\lambda\left(n S_{d-1}(\not)\right) \mathcal{K}\right)+\mathcal{G g} \text {. }
$$

But

$$
\lambda\left(S_{d-1}(p) \notin\right) \subset \lambda\left(S_{d-1}(p)\right) \notin+\lambda\left(S_{d-1}(g)\right)
$$

and

$$
\lambda\left(n S_{d-1}(p)\right) \subset n \lambda\left(S_{d-1}(p)\right)+\lambda\left(S_{d-1}(g)\right)
$$

(see [6(b), p. 193, formula (1)]). Hence

$$
y \in\left(\lambda\left(S_{d-1}(g)\right) \mathcal{K}+n \biguplus\right)+g_{0}
$$

But

$$
\lambda\left(S_{d-1}(g)\right) \subset \sum_{i+j+k \leq d-1} \lambda\left(S_{i}(n)\right) \lambda\left(S_{j}(a)\right) \lambda\left(S_{k}(\mathfrak{k})\right)
$$

(see [6(b), p. 193, Lemma 12]), so that $\operatorname{deg} P_{g}^{*}(y)<d$. Q.E.D.

Theorem 4.3. We have that $\operatorname{Ker} P_{\mathrm{g}} \mid \varrho^{K}=\mathcal{G}^{K} \cap \varrho_{g}$, and that $P_{\mathrm{g}}^{*} \mid \varrho^{K} / \varrho^{K} \cap$ @g is injective.

Proof. The first statement follows from the second, and the second follows immediately from Lemmas 4.1 and 4.2. Q.E.D.

We can now give a nonrepresentation-theoretic proof of Proposition 3.3:

Corollary 4.4 (same as Proposition 3.3). The map $P \mid \mathrm{G}^{K}$ is injective.

Proof. Take $\mathscr{I}=0$ in Theorem 4.3. Q.E.D.

Corollary 4.5. Let $\beta \in \hat{K}$. Then the kernel of the map

$$
P_{\beta}: g^{K} \rightarrow \prod_{\gamma \in \Gamma_{\beta}} \propto \otimes K^{M} / g^{\beta, \gamma}
$$

is $\mathfrak{G}^{K} \cap \mathfrak{G}^{\beta}=\mathscr{G}^{K} \cap \mathscr{g}^{\beta} \mathcal{G}$. In particular, $P_{\beta}$ induces an algebra anti-injection

$$
P_{\beta}: \mathscr{G}^{K} / \mathcal{G}^{K} \cap \varrho^{\beta} \rightarrow \prod_{\gamma \in \Gamma_{\beta}} \mathbb{A} \otimes \mathcal{K}^{M} / \mathcal{I}^{\beta, \gamma} .
$$

Proof. Take $g_{=} g^{\beta}$ in Theorem 4.3, and apply Lemma 2.5. Q.E.D. 
Re mark 4.6. Taking for $\beta$ the trivial class in Corollary 4.5, we have HarishChandra's theorem (see [6(g), p. 260, Theorem 1] and [7(a), p. 431, Lemma 6.13]) that the kernel of the classical map $P_{\beta} \mid \varrho^{K}$ (see Remark 3.4) is precisely $\oint^{K} \cap$ $\mathcal{G}^{E}=\mathcal{G}^{K} \cap £$, and hence that $P_{\beta}$ induces an algebra injection of $\mathcal{G}^{K} / \mathcal{G}^{K} \cap \mathcal{G}^{E}$ into $\mathbb{A}_{\text {; }}$ in particular, $\mathscr{G}^{K} / \mathscr{G}^{K} \cap \mathcal{G}^{K}$ is commutative.

We shall use the following in $\$ 10$ :

Lemma 4.7. Let $V$ be a vector space and let $T: \mathcal{K} \rightarrow V$ be a linear map. Define the linear maps $P_{T}: \biguplus \rightarrow S(a) \otimes V$ by

$$
P_{T}=\left(\lambda^{-1} \otimes T\right) \circ P
$$

and $f_{T}: @ \rightarrow S(a) \otimes V$ by

$$
f_{T}=(1 \otimes T) \circ F_{0}^{-1 \circ} f_{0},
$$

where $F_{0}$ and $f_{0}$ denote the above maps $F_{g}$ and $f_{g}$ in the case $g=0$. For all $x \in S(a) \otimes V$, define $\operatorname{deg} x$ to be the degree of $x$ with respect to the grading of $S(a) \otimes V$ induced by the natural grading of $S(a)$. Also, for all $x \in \mathcal{G}$, let $\operatorname{deg} x$ denote the degree of $x$ in the above sense, with $9=0$. Then for all $x \in \mathcal{G}$, we bave

$$
\operatorname{deg}\left(P_{T}(x)-f_{T}(x)\right)<\operatorname{deg} x .
$$

Moreover, if $T(1) \neq 0$, then $\left.P_{T} \mid \lambda(S(\not))^{K}\right)$ is injective (where $\left.S(k)\right)^{K}$ denotes the set of $K$-invariants in $S(\xi)$ under the natural action of $K$ on $S(p))$.

Proof. The first assertion follows immediately from Lemma 4.2 for $9=0$, since $P_{T}=(1 \otimes T) \circ F_{0}^{-1} \circ P_{0}^{*}$. Suppose that $T(1) \neq 0$, and let $\left.x \in \lambda(S(p))^{K}\right)$. Then $\operatorname{deg} f_{0}(x)=\operatorname{deg} x$ by Lemma 4.1 for $9=0$. But $f_{0}(x) \in \mathbb{P}$ and $T(1) \neq 0$, so that $\operatorname{deg} f_{T}(x)=\operatorname{deg} f_{0}(x)$. If $P_{T}(x)=0$, then $\operatorname{deg} f_{0}(x)=\operatorname{deg} f_{T}(x)<\operatorname{deg} x$ by the first assertion. Since $\operatorname{deg} f_{0}(x)=\operatorname{deg} x$, we must have $x=0$. Q.E.D.

5. The bound on multiplicities. In this section, we shall apply Corollary 4.5 to representation theory, by extending techniques of $\mathrm{R}$. Godement $[5, \S 1]$ and J. Dixmier ([3(a)] and $[3(\mathrm{~b}), \S 15.5])$ to groups $G$ without faithful finitedimensional representations. Theorem 5.4 is a sharpening of $[6(c), p .36$, Theorem 3]. Theorem 5.5 had been obtained by Harish-Chandra [6(h), Theorem 4, first assertion], by more difficult analytic methods.

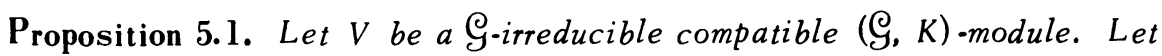
$\beta \in \hat{K}$, and let $X \in \beta$. Then

$$
\operatorname{dim} \operatorname{Hom}_{K}(X, V)=m([V], \beta),
$$

and if $V_{\beta} \neq 0$, then the $\mathcal{G}^{K}$-module structure on $\operatorname{Hom}_{K}(X, V)$ naturally induces

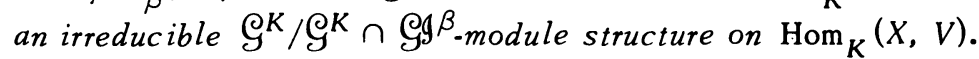


Proof. The first statement is clear, and the second statement follows from Lemma 2.1 and the remarks in $\$ 2$. Q.E.D.

Remark 5.2. Since $K$ is connected, the second statement of Proposition 5.1 also follows from [11, Theorem 5.5].

For every $\beta \in \hat{K}$, let $d(\beta)$ denote the degree of $\beta$, that is, the dimension of any module in $\beta$.

Theorem 5.3. Let $\beta \in \hat{K}$, and let $Y$ be an irreducible $\mathcal{G}^{K} / \mathcal{G}^{K} \cap \mathrm{gg}^{\beta}$-module. Then

$$
\operatorname{dim} Y \leq \max _{\gamma \in \hat{M}} m(\beta, \gamma) \quad(\leq d(\beta)<\infty)
$$

Proof. For every $\gamma \in \Gamma_{\beta}$ and $\lambda \in a^{\prime}$, let

$$
f_{\beta, \lambda}: \prod_{\gamma \in \mathbf{\Gamma}_{\beta}} \oplus \otimes K^{M} / \mathcal{I}^{\beta, \gamma} \rightarrow K^{M} / \mathcal{I}^{\beta, \gamma}
$$

denote the antihomomorphism obtained by projecting to the factor corresponding to $\gamma$, applying the evaluation homomorphism $\pi_{\lambda} \otimes 1$, and composing with the trans pose antiautomorphism of the matrix algebra $\mathcal{K}^{M} / \mathcal{I}^{\beta, \gamma}$. Then by Corollary 4.5, the homomorphisms $f_{\beta, \gamma} \circ P_{\beta}$ form a separating set of finite dimensional representations of $\mathcal{G}^{K} / \mathcal{G}^{K} \cap \mathfrak{G g}^{\beta}$ of degrees equal to or less than $\max m(\beta, \gamma)(\gamma \in \hat{M})$. By Lemma 2.2, the commuting ring of $Y$ consists of the scalars. The result now follows from the Jacobson density theorem and [5, P. 503, Lemma 1]. Q.E.D.

Theorem 5.4 (cf. [9, Lemma 1.3.2 and Remark 1.3.3]). Let $V$ be a S- $^{-}$ irreducible compatible $(\mathcal{G}, K)$-module. Then for all $\beta \in \hat{K}$,

$$
m([V], \beta) \leq \max _{\gamma \in \hat{M}} m(\beta, \gamma) \quad(\leq d(\beta)<\infty) .
$$

Proof. Theorem 5.4 follows immediately from Proposition 5.1 and Theorem 5.3. Q.E.D.

Theorem 5.5, Let $\pi$ be a continuous topologically irreducible representation of $G$ on a Banach space such that the multiplicity of any element of $\hat{K}$ is finite. Then for all $\beta \in \hat{K}$, the multiplicity of $\beta$ in $\pi$ is equal to or less than $\max m(\beta, \gamma)$ $(\gamma \in \hat{M})$ and bence equal to or less than $d(\beta)$.

Proof. By Harish-Chandra's results [6(b)], the representation of $\mathcal{G}$ on the

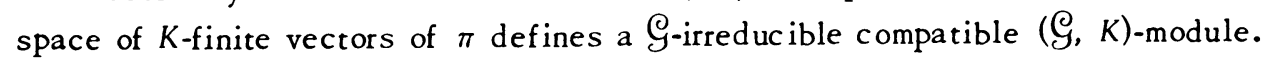
The result now follows from The orem 5.4. Q.E.D.

Remark 5.6. The (first) bound in Theorems 5.3, 5.4 and 5.5 is the best possible, in view of $\mathrm{F}$. Bruhat's result $[2, \mathrm{p} .193$, Théorème $7 ; 2 \mathrm{a}]$ concerning irreducibility of the principal series. 
6. Effective highest weight maps. In $\$ 6$, we shall further relate the mappings of $\$ 3$ to representation theory.

Definition 6.1. Let $V$ be an ( $\mathfrak{A} \gamma, M)$-module, and let $\gamma \in \hat{M}, \lambda \in a^{\prime}$. Fix an $M$-module $Z$ in the class $\gamma$, and regard $Z$ as an ( $(\mathbb{P}), M$ )-module by means of $\lambda$, the trivial action of $n$ and the given action of $M$. We call an ((P) $\pi, M$ )-module map $f: V \rightarrow Z$ a bighest weight map for $V$ with respect to $\gamma$ and $\lambda$. If $V$ is a (乌, M)-module (and hence an ( $(\mathcal{T}), M$ )-module by restriction), then a highest weight map $f$ is said to be effective if for all nonzero $v \in V$, we have

$$
f(乌 \cdot v) \neq 0 \text {. }
$$

If $V$ is a $(\mathcal{G}, K)$-module and if $\beta \in \hat{K}$, then a highest weight map $f$ is said to be effective at $\beta$ if $(*)$ holds for all nonzero $v \in V_{\beta}$.

Remark 6.2. The notion of effective highest weight map was suggested by the special case of [9, Definition 1.7.8].

Remark 6.3. In the notation of Definition 6.1 (*), $f(\varrho \cdot v)=f(\mathcal{K} \cdot v)$ for all

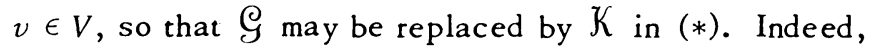

$$
f(\biguplus \cdot v)=f(\Re(\mathcal{T} K \cdot v)=\Re(\mathcal{R} \cdot f(\mathcal{K} \cdot v)=\mathbf{C} f(\mathcal{K} \cdot v)=f(\mathcal{K} \cdot v) .
$$

Proposition 6.4. Let $V$ be a compatible (乌, K)-module, and let $\beta \in \hat{K}$, $\gamma \in \hat{M}, \lambda \in a^{\prime}, X \in \beta$ and $Z \in \gamma$. Let $f: V \rightarrow Z$ be a bighest weight map with respect to $\gamma$ and $\lambda$. Then there is a natural bilinear pairing

$$
\phi: \operatorname{Hom}_{M}(Z, X) \times \operatorname{Hom}_{K}(X, V) \rightarrow \operatorname{Hom}_{M}(Z, Z) \simeq \mathbf{C}
$$

given by

$$
g, b \mapsto f \circ b \circ g
$$

for all $g \in \operatorname{Hom}_{M}(Z, X)$ and $b \in \operatorname{Hom}_{K}(X, V)$. For such $g$, $h$, and $u \in \mathcal{G}^{K}$, we bave

$$
\phi(g, u \cdot b)=\phi\left(P_{\lambda}(u) \cdot g, b\right)\left(=\phi\left(P_{\beta, \gamma, \lambda}(u) \cdot g, b\right)\right) .
$$

If $f$ is effective at $\beta$, the right kernel of $\phi$ is zero.

Proof. The first statement is clear. To prove the second, write

$$
u \equiv \sum_{i} a_{i} k_{i} \quad(\bmod n \biguplus)
$$

where $a_{i} \in \mathbb{P}$ and $k_{i} \in \mathcal{K}^{M}$. Let $\sigma$ denote the representation of $\mathcal{G}$ on $V$ and let $\tau$ denote the representation of $\mathcal{K}$ on $X$. Then

$$
\begin{aligned}
\phi(g, u \cdot b) & =f \circ(u \cdot b) \circ g=f \circ \sigma(u) \circ b \circ g \\
& =\sum_{i} \pi_{\lambda}\left(a_{i}\right) f \circ \sigma\left(k_{i}\right) \circ b \circ g=\sum_{i} \pi_{\lambda}\left(a_{i}\right) f \circ b \circ \tau\left(k_{i}\right) \circ g \\
& =f \circ b \circ P_{\lambda}(u) \cdot g=\phi\left(P_{\lambda}(u) \cdot g, b\right) .
\end{aligned}
$$


Finally, suppose that $f$ is effective at $\beta$, and suppose that $b \in \operatorname{Hom}_{K}(X, V)$, $b \neq 0$. If $f \circ b=0$, then

$$
f(\mathcal{K} \cdot b(X))=f(b(\mathcal{K} \cdot X))=0,
$$

contradicting the effectiveness of $f$, in view of Remark 6.3. Hence $f \circ b \neq 0$, and so there exists $g \in \operatorname{Hom}_{M}(Z, X)$ such that $\phi(g, b)=f \circ b \circ g \neq 0$. Q.E.D.

Theorem 6.5. Let $V$ be a compatible (乌, K)-module which admits a bighest weight map $f$ with respect to $\gamma \in \hat{M}$ and $\lambda \in a^{\prime}$, such that $f$ is effective at $\beta \in \hat{K}$. Then $m([V], \beta) \leq m(\beta, \gamma)(<\infty)$, and if $m(\beta, \gamma)>0$, then $\operatorname{Ker} \eta([V], \beta)$ $\supset \operatorname{Ker} P_{\beta, \gamma, \lambda} \mid \mathcal{G}^{K}$. Suppose that $m([V], \beta)=m(\beta, \gamma)>0$. Then

and

$$
\operatorname{Ker} \eta([V], \beta)=\operatorname{Ker} P_{\beta, \gamma, \lambda} \mid \varrho^{K} \text {, }
$$

$$
\chi(\eta([V], \beta))=p_{\beta, \gamma, \lambda} \mid \biguplus^{K} .
$$

Moreover, let $X \in \beta$ and $Z \in \gamma$, and assume that $f: V \rightarrow Z$. Let $\omega$ denote the representation of $\mathcal{G}^{K}$ on $\operatorname{Hom}_{K}(X, V)$. Then

$$
\begin{gathered}
\phi: \operatorname{Hom}_{M}(Z, X) \times \operatorname{Hom}_{K}(X, V) \rightarrow \operatorname{Hom}_{M}(Z, Z) \simeq \mathrm{C}, \\
g, \quad b \mapsto f \circ b \circ g
\end{gathered}
$$

is a nonsingular pairing, and for all $u \in \mathcal{G}^{K}, \omega(u)$ and $P_{\beta, \gamma, \lambda}(u)$ are adjoint to each other with respect to $\phi$.

Proof. All the assertions follow easily from Proposition 6.4. Q.E.D.

Remark 6.6. Let $V$ be a finite-dimensional irreducible $G$-module. Then it is well known that the lowest restricted weight space $Z$ of $V$ is $M$-irreducible, giving rise to some $\gamma \in \hat{M}$. Let $\lambda \in a^{\prime}$ denote the lowest restricted weight of $V$. Then the projection of $V$ onto $Z$ with respect to the restricted weight space decomposition of $V$ is an effective highest weight map for $V$ with respect to $\gamma$ and $\lambda$, so that Theorem 6.5 applies.

Remark 6.7. Theorem 6.5 may be regarded as a generalization of [6(c), p. 49, Lemma 11],[12, Theorem 2.2 (2.34)] (cf. Remarks 3.5 and 6.6) and [9, Lemma 1.7.6]).

7. The nonunitary principal series. In this section, we shall construct a series of representations admitting effective highest weight maps, so that the results of $\$ 6$ may be applied. The representations are essentially those of the classical nonunitary principal series.

The exponential map exp: $a_{\mathrm{R}} \rightarrow A$ is an analytic diffeomorphism; let us denote its inverse by log. Since every differentiable finite-dimensional 
irreducible $A$-module is one-dimensional, we may identify $\hat{A}$ with $a^{\prime}$, under the exponential map. Let $\rho \in \hat{A}\left(=a^{\prime}\right)$ denote half the sum of the position roots (of $g$ with respect to $a)$, with multiplicities counted.

Fix $\gamma \in \hat{M}, \lambda \in \hat{A}$, and $Z \in \gamma$. Define the following $M A N$-module structure on $Z$ :

$$
\operatorname{man} \cdot z=(i \lambda+\rho)(a) m \cdot z \quad\left(=e^{(i \lambda+\rho)(\log a)} m \cdot z\right)
$$

for all $m \in M, a \in A, n \in N$ and $z \in Z\left(i=(-1)^{1 / 2}\right)$. (The reason for the expression $i \lambda+\rho$ will become apparent in the following sections.)

Let $V^{(\gamma, \lambda)}$ be the space of all analytic functions $f: G \rightarrow Z$ such that

$$
f(g \text { man })=(\text { man })^{-1} \cdot f(g)=e^{-(i \lambda+\rho)(\log a)} m^{-1} \cdot f(g)
$$

for all $g \in G, m \in M, a \in A$ and $n \in N$. Then $V^{(\gamma, \lambda)}$ is a $G$-module under the action given by $(g \cdot f)(b)=f\left(g^{-1} b\right)$ for all $f \in V^{(\gamma, \lambda)}$ and $g, b \in G$. Furthermore, $V^{(\gamma, \lambda)}$ is a $g_{R}$-module, and hence a $g$-module and a $\oint_{\text {-module, under the action }}$ given by

$$
(x \cdot f)(g)=\left.\frac{d}{d t}((\exp t x) \cdot f)(g)\right|_{t=0}=\left.\frac{d}{d t} f((\exp -t x) g)\right|_{t=0}
$$

for all $f \in V^{(\gamma, \lambda)}, x \in g_{\mathrm{R}}$ and $g \in G$. Then $V^{(\gamma, \lambda)}$ is a $(\mathcal{G}, G)$-module.

Let $V^{\gamma, \lambda}$ denote the subspace of $V^{(\gamma, \lambda)}$ consisting of the $K$-finite vectors.

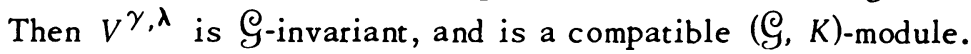

Let $U^{(\gamma)}$ be the space of all analytic functions $f: K \rightarrow Z$ such that $f(k m)$ $=m^{-1} \cdot f(k)$ for all $k \in K$ and $m \in M$. Then $U^{(\gamma)}$ is a $K$-module under the action given by $(k \cdot f)(l)=f\left(k^{-1} l\right)$ for all $f \in U^{(\gamma)}$ and $k, l \in K$. Moreover, $U^{(\gamma)}$ is a $(\mathcal{K}, K)$-module under the action of $\mathcal{K}$ determined by the following action of $\mathrm{t}_{\mathrm{R}}$ :

$$
(x \cdot f)(k)=\left.\frac{d}{d t}((\exp t x) \cdot f)(k)\right|_{t=0}=\left.\frac{d}{d t} f((\exp -t x) k)\right|_{t=0}
$$

for all $f \in U^{(\gamma)}, x \in E_{\mathrm{R}}$ and $k \in K$.

Let $U^{\gamma}$ denote the space of $K$-finite vectors in $U^{(\gamma)}$. Then $U^{\gamma}$ is $K$ invariant, and is a compatible $(\mathcal{K}, K)$-module.

Proposition 7.1 (cf. [9, Lemma 1.7.3]). The restriction map $R^{\gamma, \lambda}: V^{\gamma, \lambda}$ $\rightarrow U^{\gamma}$ is a $(\mathcal{K}, K)$-module isomorphism whose inverse is given as follows:

$$
\left(\left(R^{\gamma, \boldsymbol{\lambda}}\right)^{-1}(f)\right)(k a n)=e^{-(i \boldsymbol{\lambda}+\rho)(\log a)} f(k)
$$

for all $f \in U^{\gamma}, k \in K, a \in A$ and $n \in N$. Moreover, for all $\beta \in \hat{K}$, we have $m\left(\left[U^{\gamma}\right], \beta\right)=m(\beta, \gamma)$, so that in particular, $m\left(\left[V^{\gamma, \lambda}\right], \beta\right)=m(\beta, \gamma)$. 
Proof. The proof of the first part of the proposition is straightforward, and the second part of the proposition follows from the Frobenius reciprocity theorem. Q.E.D. of $G$.

Let $\delta_{\gamma, \lambda}: V^{\gamma, \lambda} \rightarrow Z$ denote the evaluation map at the identity element $e$

Theorem 7.2 (cf. [9, Lemma 1.7.5]). We have that $\delta_{\gamma, \lambda}$ is an effective bighest weight map for $V^{\gamma, \lambda}$ with respect to $\gamma$ and $i \lambda+\rho$ (regarding $Z$ as an $(\mathfrak{A}), M)$-module by means of its structure as an MAN-module).

Proof. Let $f \in V^{\gamma, \lambda}$ and $x \in a_{\mathrm{R}}$. Then

$$
\begin{aligned}
\delta_{\gamma, \lambda}(x \cdot f) & =(x \cdot f)(e) \\
& =\left.\frac{d}{d t} f(\exp -t x)\right|_{t=0}=\left.\frac{d}{d t} e^{t(i \lambda+\rho)(x)} f(e)\right|_{t=0} \\
& =(i \lambda+\rho)(x) f(e)=(i \lambda+\rho)(x) \delta_{\gamma, \lambda}(f),
\end{aligned}
$$

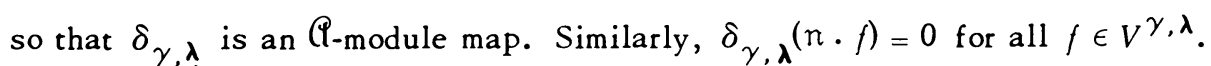
Let $f \in V^{\gamma, \lambda}$ and $m \in M$. Then

$$
\delta_{\gamma, \lambda}(m \cdot f)=(m \cdot f)(e)=f\left(m^{-1}\right)=m \cdot f(e)=m \cdot \delta_{\gamma, \lambda}(f),
$$

so that $\delta_{\gamma, \lambda}$ is an $M$-module map and hence a highest weight map.

Finally, suppose that $\delta_{\gamma, \lambda}(\mathcal{K} \cdot f)=0$ for some $f \in V^{\gamma, \lambda}$. Then $\delta_{\gamma, \lambda}(K \cdot f)$ $=0$, and so $f(k)=0$ for all $k \in K$. Thus $f=0$ by Proposition 7.1, so that $\delta_{\gamma, \lambda}$ is effective. Q.E.D.

By Theorem 6.5, Proposition 7.1 and Theorem 7.2, we now have

Theorem 7.3. Let $\gamma \in \hat{M}, \lambda \in \hat{A}$ and $\beta \in \hat{K}$ such that $m(\beta, \gamma)>0$. Then

$$
\operatorname{Ker} \eta\left(\left[V^{\gamma, \lambda}\right], \beta\right)=\operatorname{Ker} P_{\beta, \gamma, i \lambda+\rho} \mid \varrho^{K}
$$

and

$$
\chi\left(\eta\left(\left[V^{\gamma, \lambda}\right], \beta\right)\right)=p_{\beta, \gamma, i \lambda+\rho} \mid \varrho^{K} .
$$

Remark 7.4. The second formula in Theorem 7.3 will be generalized by Theorem 9.8(2) below.

Remark 7.5. The second formula in Theorem 7.3 may be regarded as a generalization of [12, Theorem 2.3 (2.55)] (cf. Remarks 3.5 and 6.7) and of [9, Lemma 1.7.5]; cf. also [6(c), p. 49, Lemma 11].

Let $\gamma \in \hat{M}, \lambda \in \hat{A}$ and $Z \in \gamma$. Regard $Z$ as a Hilbert space, with inner product $(\cdot, \cdot)$, on which the action of $M$ is unitary. A measurable function $f$ : $K \rightarrow Z$ is said to be square integrable if 


$$
\int_{K}(f(k), f(k)) d k<\infty,
$$

where $d k$ denotes normalized Haar measure on $K$. Let $H^{\gamma, \lambda}$ denote the set of equivalence classes (identifying functions whose restrictions to $K$ differ on a set of measure zero) of functions $f: G \rightarrow Z$ which are measurable and square integrable on $K$ and such that

$$
f(g \text { man })=e^{-(i \lambda+\rho)(\log a)} m^{-1} \cdot f(g)
$$

for all $g \in G, m \in M, a \in A$ and $n \in N$. Define an inner product $(\cdot, \cdot)$ in $H^{\gamma, \lambda}$ by

$$
\left(f_{1}, f_{2}\right)=\int_{K}\left(f_{1}(k), f_{2}(k)\right) d k
$$

Then $H^{\gamma, \lambda}$ is a Hilbert space, and the action given by $\left(\pi^{\gamma, \lambda}(g)(f)\right)(b)=f\left(g^{-1} b\right)$ $\left(f \in H^{\gamma, \lambda}, g, b \in G\right)$ defines a continuous (not necessarily unitary) representation $\pi^{\gamma, \lambda}$ of $G$ on $H^{\gamma, \lambda}$. Moreover, $V^{\gamma, \lambda}$ is the $乌$-module of $K$-finite vectors of $H^{\gamma, \lambda}$

The representations $\pi^{\gamma, \lambda}$ constitute the nonunitary principal series of $G$, and the $(\varrho, K)$-modules $V^{\gamma, \lambda}$ constitute the infinitesimal nonunitary principal series.

8. The subquotient theorem. A subquotient of a module $V$ is by definition a module of the form $Y / X$, where $X$ and $Y$ are submodules of $V$ such that $X \subset$ $Y$. This applies for example to $(\mathcal{G}, K)$-modules, to $\mathcal{G}^{K} / \mathscr{G}^{K} \cap \mathfrak{G g}^{\beta}$-modules $(\beta \in \hat{K})$ or to continuous representations of $G$ on a Banach space; in the last case, we require that $X$ and $Y$ be closed subspaces of the Banach space $V$. In this section, we show that various types of irreducible modules can be realized as subquotients of certain concrete modules, extending and simplifying Harish-Chandra's results [6(c), p. 63, Theorem 4] and [6(h), Theorem 4]. Our proof is essentially patterned after Harish-Chandra's proof in [6(c)], except for our use of Corollary 4.5 and Theorem 5.3 in place of Harish-Chandra's [6(c), p. 28, Lemma 1] and [6(b), p. 195, Theorem 1], respectively.

Let $G_{m}$ be a Cartan subalgebra of the complexified Lie algebra $m$ of $M$, so that $\mathfrak{G}=\mathfrak{h}_{\mathfrak{m}}+a$ is a Cartan subalgebra of $g$. Choose a system of positive roots of $m$ with respect to $\mathfrak{h}_{\mathfrak{m}}$, and let $n_{\mathfrak{m}}$ be the sum of the positive root spaces in $m$. Then $n_{+}+n_{m}$ is the sum of the positive root spaces for a certain system of positive roots of $g$ with respect to $h$, which we fix. Let $\mathbb{M}, \mathcal{H}_{m}$ and $\mathcal{H}$ be the universal enveloping algebras of $m, \mathfrak{h}_{m}$ and $\mathfrak{h}$, respectively, regarded as canonically embedded in $乌$. Let $\mathcal{Z}_{\mathrm{S}}$ be the center of $\mathcal{G}_{\text {. }}$

Let $W_{\mathfrak{h}}$ be the Weyl group of $g$ with respect to $\mathfrak{h}$, regarded as acting on $\mathfrak{b}^{\prime}$, and let $\rho_{n} \in \mathfrak{G}^{\prime}$ be half the sum of the positive roots of $g$ with respect to $h$. For all $s \in W_{\mathfrak{b}}$, let $s_{*}$ denote the affine transformation of $\mathscr{G}^{\prime}$ given by 


$$
s * \nu=s\left(\nu-\rho_{\mathfrak{h}}\right)+\rho_{\mathfrak{h}}
$$

for all $\nu \in \mathfrak{G}^{\prime}$. Let $s^{*}$ denote the algebra automorphism of $\mathcal{H}$ (identified with the algebra of polynomial functions on $b^{\prime}$ ) given by

$$
\left(s^{*} x\right)(\nu)=x\left(s_{*}^{-1} \nu\right)
$$

for all $x \in \mathcal{H}, \nu \in \mathfrak{G}^{\prime}$. We obtain an action

$$
W_{\mathfrak{h}} \rightarrow W_{\mathfrak{h}}^{*}=\left\{s^{*} \mid s \in W_{\mathfrak{h}}\right\}
$$

of $W_{\mathfrak{b}}$ as automorphisms of $\mathcal{H}$. Let $\mathcal{H}^{*}$ denote the set of $W_{\mathfrak{h}}$-invariants in $\mathcal{H}$ with respect to this action.

For all subsets $S, T \subset \mathcal{G}$, let $T^{S}$ denote the centralizer of $S$ in $T$. For all $\nu \in \mathfrak{G}^{\prime}$, let $\pi_{\nu}: \mathcal{H} \rightarrow \mathrm{C}$ be the evaluation homomorphism at $\nu$.

The following lemma, due to Harish-Chandra, is well known, and we omit the proof:

Lemma 8.1 (see [6(f), p. 118, Lemmas 18 and 19]). We bave $\mathcal{G}^{\mathfrak{h}} \subset \mathcal{H}+$ $\left(n+n_{m}\right) \mathcal{G}$ (direct sum). Denoting by $\chi^{\mathfrak{B}}: \mathcal{G}^{\mathfrak{h}} \rightarrow \mathcal{H}$ the corresponding projection

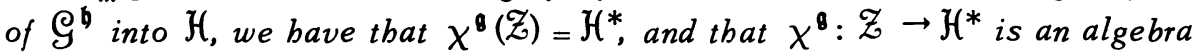
isomorphism. For all $\nu \in \mathfrak{G}^{\prime}$, let

$$
\chi_{\nu}^{g}=\pi_{\nu}^{\circ} \chi^{g}: 乌^{\mathfrak{h}} \rightarrow \mathbf{C} .
$$

Then every bomomorphism from $\mathcal{Z}$ into $\mathbf{C}$ is of the form $\chi_{\nu}^{8} \mid \mathcal{Z}$ for some $\nu \in \mathfrak{G}^{\prime}$.

The proof of the following lemma is straightforward:

Lemma 8.2. We bave $\mathbb{N}^{\mathfrak{h} m} \subset \mathcal{H}_{m}+n_{m} M$ (direct sum), giving rise to the projection map $\chi^{\mathrm{m}}: \mathfrak{\pi}^{\mathfrak{b}_{\mathfrak{m}}} \rightarrow \mathcal{H}_{\mathrm{m}}$. Moreover,

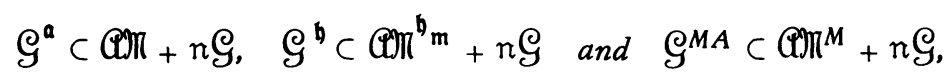

so that

$$
P\left(\bigodot^{\mathfrak{a}}\right) \subset \mathbb{Q} \otimes \mathbb{M}, \quad P\left(\bigodot^{\mathfrak{b}}\right) \subset \mathbb{Q} \otimes \mathbb{M}^{\mathfrak{b} m} \text { and } P\left(\bigodot^{M A}\right) \subset \mathbb{Q} \otimes \mathbb{M}^{M} .
$$

Finally,

$$
\chi^{8}=\left(1 \otimes \chi^{\mathfrak{m}}\right) \circ P: \mathfrak{S}^{\mathfrak{h}} \rightarrow \mathcal{H}
$$

(identifying $\mathcal{H}$ with $\mathscr{Q} \otimes \mathcal{H}_{\mathfrak{m}}$ ).

For all $\mu \in \mathfrak{G}_{\mathfrak{m}}^{\prime}$, let $\pi_{\mu}: \mathcal{H}_{\mathfrak{m}} \rightarrow \mathbf{C}$ denote the evaluation homomorphism at $\mu$. For all $\gamma \in \hat{M}$, let $\mu_{\gamma} \in \mathfrak{G}_{m}^{\prime}$ denote the lowest weight of the m-module(3) induced by any module in the class $\gamma$. Also, let

$$
\chi_{\gamma}^{8}=\left(1 \otimes \pi_{\mu}\right) \circ \chi^{8}: 乌^{\mathfrak{h}} \rightarrow \mathfrak{Q}
$$

(3) It is known that this m-module is primary but not necessarily irreducible. 
(identifying $\mathcal{H}_{\text {with }} \oplus \otimes \mathcal{H}_{m}$ ), and for all $\lambda \in a^{\prime}$, let

$$
\chi_{\gamma, \lambda}^{8}=\left(\pi_{\lambda} \otimes \pi_{\mu_{\gamma}}\right) \circ \chi^{8}: \mathcal{G}^{\mathfrak{b}} \rightarrow \mathbf{C}
$$

(identifying $\mathcal{H}$ with $\left.\mathscr{P} \otimes \mathcal{H}_{m}\right)$.

Lemma 8.3. Let $\beta \in \hat{K}$ and $\gamma \in \hat{M}$ such that $m(\beta, \gamma)>0$. Then

$$
P_{\beta, \gamma}\left(\mathcal{G}^{M A}\right) \subset \mathbb{A} \subset \oplus \otimes K^{M} / \mathscr{I}^{\beta, \gamma}
$$

(identifying $($ with $\propto \otimes 1$ ),

$$
P_{\beta, \gamma}=\chi_{\gamma}^{8}: \varrho^{M A} \rightarrow \mathbb{A},
$$

and for all $\lambda \in a^{\prime}$,

$$
P_{\beta, \gamma, \boldsymbol{\lambda}}=\chi_{\gamma, \boldsymbol{\lambda}}^{\mathrm{B}}: \mathrm{S}^{M A} \rightarrow \mathbf{C} .
$$

Proof. We recall from $\$ 3$ the projection $\pi_{\beta, \gamma}: K^{M} \rightarrow K^{M} / \mathcal{G}^{\beta, \gamma}$. The restriction of $\pi_{\beta, \gamma}$ to $\mathbb{\pi}^{M}$ is the homomorphism from $\pi^{M}$ to $C$ by which $\mathbb{M}^{M}$ acts as scalars on an irreducible $M$-module in the class $\gamma$, and a standard argument shows that we therefore have

$$
\left.\pi_{\beta, \gamma}\right|^{M^{M}=\pi_{\mu}}{ }^{\circ} \chi^{m}: \mathbb{M}^{M} \rightarrow \mathrm{C} .
$$

Hence

$$
P_{\beta, \gamma}\left(\varrho^{M A}\right)=\left(\left(1 \otimes \pi_{\beta, \gamma}\right) \circ P\right)\left(\biguplus^{M A}\right) \subset \mathbb{Q}
$$

by Lemma 8.2 , and

$$
\begin{aligned}
P_{\beta, \gamma} \mid \varrho^{M A} & =\left(1 \otimes \pi_{\beta, \gamma}\right) \circ P\left|\varrho^{M A}=\left(1 \otimes\left(\pi_{\mu} \circ \chi^{m}\right)\right) \circ P\right| \varrho^{M A} \\
& =\left(1 \otimes \pi_{\mu_{\gamma}}\right) \circ\left(1 \otimes \chi^{m}\right) \circ P\left|\varrho^{M A}=\left(1 \otimes \pi_{\mu_{\gamma}}\right) \circ \chi^{\otimes}\right| \biguplus^{M A}
\end{aligned}
$$

(by Lemma 8.2)

$$
=\chi_{y}^{8} \mid \Theta^{M A} \text {. }
$$

The last assertion of the lemma follows immediately. Q.E.D.

Fix $\beta \in \hat{K}$ and $a \in \mathcal{G}^{K}$, so that $P_{\beta, \gamma}(a) \in \mathbb{Q} \otimes K^{M} / \mathcal{G}^{\beta, \gamma}$ for all $\gamma \in \Gamma_{\beta}$. Consider the ring $\mathbb{A}[T]$ of polynomials in one variable with coefficients in $\mathbb{Q}$. Define $f(T) \in \mathbb{P}[T]$ by

$$
f(T)=\prod_{\gamma \in \mathbf{\Gamma}_{\beta}} \operatorname{det}\left(T \cdot 1_{\beta, \gamma}-P_{\beta, \gamma}(a)\right),
$$

where $1_{\beta, \gamma}$ denotes the identity element of $\left(\mathcal{Q} \otimes \mathcal{K}^{M} / g^{\beta, \gamma}\right.$, and det denotes the 
determinant function on $\mathfrak{A}[T] \otimes K^{M} / \mathcal{I}^{\beta, \gamma}$, which is isomorphic to the algebra of $m(\beta, \gamma) \times m(\beta, \gamma)$ matrices with coefficients in $\mathbb{A}[T]$. Then $f\left(P_{\beta, \gamma}(a)\right)=0$ for all $\gamma \in \Gamma_{\beta}$.

For all $\lambda \in a^{\prime}$, let $f_{\lambda}(T)$ denote the element of the polynomial ring $\mathbf{C}[T]$ obtained by evaluating the coefficients of $f(T)$ at $\lambda$. Then

$$
f_{\lambda}(T)=\prod_{\gamma \in \Gamma_{\beta}} \operatorname{det}\left(T \cdot 1_{\beta, \gamma}-P_{\beta, \gamma, \lambda}(a)\right) .
$$

Regard $\mathfrak{Q} \subset \mathcal{H}$ in the natural way, so that $\mathbb{Q}[T] \subset \mathcal{H}[T]$. Define $\bar{f}(T) \in \mathcal{H}[T]$ by

$$
\bar{f}(T)=\prod_{s \in W_{\mathfrak{h}}} s^{*} f(T)
$$

where the action of $W_{\mathfrak{b}}^{*}$ is extended from $\mathcal{H}$ to $\mathcal{H}[T]$ in the obvious way. Then $\bar{f}(T) \in \mathcal{H}^{*}[T]$, and so Lemma 8.1 implies that there exist $z_{0}, \ldots, z_{q-1} \in Z(q=$ degree $\bar{f}(T))$ such that

$$
\bar{f}(T)=T^{q}+\chi^{8}\left(z_{q=1}\right) T^{q-1}+\cdots+\chi^{8}\left(z_{1}\right) T+\chi^{8}\left(z_{0}\right) .
$$

Let

$$
b=a^{q}+z_{q-1} a^{q-1}+\cdots+z_{1} a+z_{0} \in \mathcal{G}^{K} .
$$

For all $\nu \in \mathfrak{G}^{\prime}$, let $\bar{f}_{\nu}(T)$ denote the element of $\mathrm{C}[T]$ obtained by evaluating the coefficients of $\vec{f}(T)$ at $\nu$. Then

$$
\bar{f}_{\nu}(T)=\prod_{s \in W_{\mathfrak{h}}} f_{s_{*}^{-1}(\nu) \mid \mathfrak{a}}(T)=\prod_{\gamma \in \Gamma_{\beta} ; s \in W_{\mathfrak{h}}} \operatorname{det}\left(T \cdot 1_{\beta, \gamma}-P_{\beta, \gamma, s_{*}^{-1}(\nu) \mid \mathfrak{a}}(a)\right)
$$

Let

$$
\mathcal{L}_{\beta, \nu}=\bigcap_{\gamma \in \Gamma_{\beta} ; s \in W_{\mathfrak{h}}} \operatorname{Ker} P_{\beta, \gamma, s_{*}^{-1}(\nu) \mid a} \mid \biguplus^{K},
$$

a two-sided ideal of $\mathcal{G}^{K}$. By the above, if $a \in \mathscr{L}_{\beta, \nu}$, then $\widetilde{f}_{\nu}(T)=T^{q}$.

Lemma 8.4. We bave that $b \in \mathcal{G}^{K} \cap \mathrm{gg}^{\beta}$

Proof. Let $\gamma \in \Gamma_{\beta}$. Then

$$
P_{\beta, \gamma^{(b)}}=P_{\beta, \gamma}(a)^{q}+P_{\beta, \gamma}\left(z_{q=1}\right) P_{\beta, \gamma}(a)^{q=1}+\cdots+P_{\beta, \gamma}\left(z_{0}\right)
$$

$$
=P_{\beta, \gamma}(a)^{q}+\chi_{\gamma}^{8}\left(z_{q-1}\right) P_{\beta, \gamma}(a)^{q-1}+\cdots+\chi_{\gamma}^{8}\left(z_{0}\right),
$$

by Proposition 3.1 and Lemma 8.3. Let 


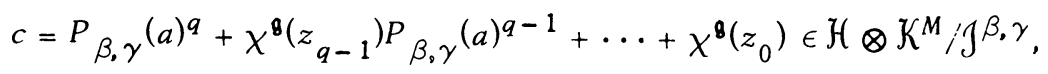

and consider the map

$$
1 \otimes \pi_{\mu \gamma} \otimes 1: \mathcal{H} \otimes K^{M} / g^{\beta, \gamma}=\mathscr{Q} \otimes \mathcal{H}_{m} \otimes K^{M} / \mathcal{I}^{\beta, \gamma} \rightarrow \mathbb{A} \otimes K^{M} / \mathcal{I}^{\beta, \gamma} .
$$

Then $(*)$ equals $\left(1 \otimes \pi_{\mu_{\gamma}} \otimes 1\right)(c)$. But

$$
c=\bar{f}\left(P_{\beta, \gamma}(a)\right)=\prod_{s \in W_{\mathfrak{h}}} s^{*} f\left(P_{\beta, \gamma}(a)\right)=0
$$

since $f\left(P_{\beta, \gamma}(a)\right)=0$. Hence $P_{\beta, \gamma}(b)=0$. Since this holds for all $\gamma \in \Gamma_{\beta}$, we have $P_{\beta}(b)=0$, and so $b \in \mathcal{G}^{K} \cap \mathrm{g}^{\beta} \beta$ by Corollary 4.5. Q.E.D.

Lemma 8.5. Let $Y$ be an irreducible $\varrho^{K} / \mathcal{G}^{K} \cap \mathrm{G}^{\beta}$-module, and regard $Y$ as a $\mathcal{G}^{K}$-module. Then $\mathcal{Z}$ acts on $Y$ according to a scalar bomomorphism of the form $\chi_{\nu}^{\mathfrak{g}} \mid \mathcal{Z}$ for some $\nu \in \mathfrak{G}^{\mathfrak{\prime}}, \operatorname{dim} Y<\infty$ and every element of $\mathfrak{L}_{\beta, \nu}$ annibilates $Y$.

Proof. $\mathscr{Z}$ acts as scalars on $Y$ by Lemma 2.2, and the existence of $\nu$ follows from Lemma 8.1. Let $\sigma: \mathcal{G}^{K} \rightarrow$ End $Y$ be the representation of $\mathcal{G}^{K}$ associated with $Y$. Since $b \in \varrho^{K} \cap \varrho^{\beta}$ by Lemma 8.4 , we have

$$
\begin{aligned}
0 & =\sigma(b)=\sigma(a)^{q}+\sigma\left(z_{q-1}\right) \sigma(a)^{q-1}+\cdots+\sigma\left(z_{1}\right) \sigma(a)+\sigma\left(z_{0}\right) \\
& =\sigma(a)^{q}+\chi_{\nu}^{8}\left(z_{q-1}\right) \sigma(a)^{q-1}+\cdots+\chi_{\nu}^{8}\left(z_{1}\right) \sigma(a)+\chi_{\nu}^{8}\left(z_{0}\right)=\bar{f}_{\nu}(\sigma(a)) .
\end{aligned}
$$

If $a \in \mathfrak{S}_{\beta, \nu}$, then we get $0=\sigma(a)^{q}$ from the above discussion. Thus every element of the two-sided ideal $\sigma\left(\varrho_{\beta, \nu}\right)$ of $\sigma\left(\varrho^{K}\right)$ is nilpotent. But $\operatorname{dim} Y<\infty$, by Theorem 5.3. Hence $\sigma\left(\varrho^{K}\right)$ is the finite-dimensional simple ring End $Y$, so that $\sigma\left(\varrho_{\beta, \nu}\right)$ $=0$. Q.E.D.

We omit the proof of the following standard fact (see [6(c), p. 56, Lemma 14]):

Lemma 8.6. Let $\mathfrak{B}$ be an algebra over $\mathrm{C}$, let $\sigma_{0}, \sigma_{1}, \ldots, \sigma_{n}$ be finitedimensional representations of $B$ and suppose that $\sigma_{0}$ is irreducible. If $\bigcap_{i=1}^{n} \operatorname{Ker} \sigma_{i} \subset \operatorname{Ker} \sigma_{0}$, then $\operatorname{Ker} \sigma_{i} \subset \operatorname{Ker} \sigma_{0}$ for some $i=1, \cdots, n$.

Theorem 8.7. Let $\beta \in \hat{K}$, and let $Y$ be an irreducible $\left(\mathcal{G}^{K} / \mathcal{G}^{K} \cap \mathcal{G}^{\beta}\right)$-module. Then there exist $\gamma \in \hat{M}$ and $\lambda \in a^{\prime}$ such that $m(\beta, \gamma)>0$ and such that $Y$ is equivalent to a subquotient of a (finite-dimensional) $\left(\mathrm{g}^{K} / \mathrm{g}^{K} \cap \mathrm{g}^{\beta}\right)$-module in the class $\eta\left(\left[V^{\gamma, \lambda}\right], \beta\right)$. In particular, $\operatorname{dim} Y \leq m(\beta, \gamma) \leq d(\beta)$ (cf. Theorem 5.3). Denoting by $\sigma: \mathrm{G}^{K} \rightarrow$ End $Y$ the representation of $\mathcal{G}^{K}$ associated with $Y$, we have $\operatorname{Ker} P_{\beta, \gamma, i \lambda_{+} \rho} \mid \mathscr{G}^{K} \subset \operatorname{Ker} \sigma$, and there exists an antibomomorphism

$$
\sigma^{\prime}: P_{\beta, \gamma, i \lambda+\rho}\left(\varrho^{K}\right) \rightarrow \text { End } Y
$$

such that $\sigma=\sigma^{\prime} \circ P_{\beta, \gamma, i \lambda_{+} \rho} \mid \oint^{K}$. Moreover, $\sigma(\mathcal{Z})$ acts on $Y$ according to the scalar homomorphism $\chi_{\gamma, i \lambda+\rho}^{\mathbf{g}} \mid \mathcal{Z}$. 
Proof. By Lemma 8.5, there exists $\nu \in \zeta^{\prime}$ such that $\sigma(\mathscr{Z})$ acts on $Y$ according to the scalar homomorphism $\chi_{\nu}^{g} \mid \mathcal{Z}$, and $\mathscr{L}_{\beta, \nu} \subset \operatorname{Ker} \sigma$. For each $\gamma \in \Gamma_{\beta}$ and $\lambda \in a^{\prime}, P_{\beta, \gamma, \lambda}$ is an antihomomorphism of $\mathcal{G}^{K}$ into a finite-dimensional matrix algebra. Hence the composition of $P_{\beta, \gamma, \lambda}$ with the transpose antiautomorphism of this matrix algebra is a finite-dimensional representation of $\oint^{K}$, and the kernel of this representation is the kernel of $P_{\beta, \gamma, \lambda}$. Thus by Lemma 8.6 and the definition of $\mathfrak{L}_{\beta, \nu}$, there exist $\gamma \in \Gamma_{\beta}$ and $s \in W_{\mathfrak{h}}$ such that $\operatorname{Ker} P_{\beta, \gamma, s_{*}^{-1}(\nu) \mid \mathfrak{a}} \mid \mathcal{G}^{K} C$ Ker $\sigma$. Choosing $\lambda \in a^{\prime}$ so that $i \lambda+\rho=s_{*}^{-1}(\nu) \mid a$, we have

$$
\operatorname{Ker} P_{\beta, \gamma, i \lambda+\rho} \mid 乌^{K} \subset \operatorname{Ker} \sigma \text {, }
$$

and the existence of the antihomomorphism $\sigma^{\prime}$ is clear. By Theorem 7.3, we have Ker $\eta\left(\left[V^{\gamma, \lambda}\right], \beta\right) \subset \operatorname{Ker} \sigma$. Let

$$
X=X_{0} \supset X_{1} \supset \cdots \supset X_{n}=0
$$

be a composition series of a $\oint^{K}$-module $X$ in the class $\eta\left(\left[V^{\gamma, \lambda}\right], \beta\right)$, so that $X_{i} / X_{i+1}$ is an irreducible $\mathcal{G}^{K}$-module for all $i=0, \ldots, n-1$. Then for all $x \in$ $\cap_{i=0}^{n-1} \operatorname{Ker}\left[X_{i} / X_{i+1}\right]$, we have

$$
x^{n} \in \operatorname{Ker} \eta\left(\left[V^{\gamma, \lambda}\right], \beta\right) \subset \operatorname{Ker} \sigma,
$$

so that $\sigma\left(\bigcap_{\operatorname{Ker}}\left[X_{i} / X_{i+1}\right]\right)$ is a two-sided ideal of $\sigma\left(\mathcal{Y}^{K}\right)$ each of whose elements is nilpotent. Since $\sigma$ is irreducible, $\sigma\left(\mathcal{G}^{K}\right)=\operatorname{End} Y$, and so $\sigma\left(\bigcap \operatorname{Ker}\left[X_{i} / X_{i+1}\right]\right)=$ 0 . Hence $Y$ (regarded as a $\mathcal{G}^{K}$-module) is equivalent to $X_{i} / X_{i+1}$ for some $i=$ $0, \ldots, n-1$, by Lemma 8.6, proving the first assertion of the theorem, and hence the second. Finally, the last statement of the theorem follows from the last statement of Lemma 8.3 and the existence of $\sigma^{\prime}$. Q.E.D.

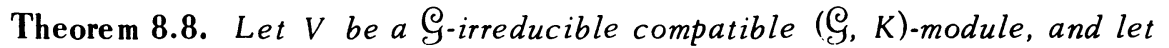
$\beta \in \hat{K}$ such that $m([V], \beta)>0$. Then there exist $\gamma \in \hat{M}$ and $\lambda \epsilon a^{\prime}$ such that $m(\beta, \gamma)>0$ and such that $V$ is equivalent to a subquotient of the $(\Im, K)$-module $V^{\gamma, \lambda}$. In particular, $m(\lceil V\rceil, \beta) \leq m(\beta, \gamma) \leq d(\beta)$ (cf. Theorem 5.4). Moreover, Z acts on $V$ according to the scalar bomomorphism $\chi_{\gamma_{, i \lambda+\rho}^{\mathfrak{g}}} \mid \mathcal{Z}$.

Proof. Let $X \in \beta$, and let $Y=\operatorname{Hom}_{K}\left(X, V_{\beta}\right)$. Then $Y$ is an irreducible $\varrho^{K}$-module, by Lemma 2.1 , and $Y$ may be regarded as an irreducible $\left(\varrho^{K} / \varrho^{K} \cap\right.$ $\oint^{\beta}{ }^{\beta}$-module. Choose $\gamma \in \hat{M}$ and $\lambda \in a^{\prime}$ as in Theorem 8.7 , so that $Y$ is equivalent to a subquotient of $\operatorname{Hom}_{K}\left(X, V^{\gamma, \lambda}\right)$. Now $V_{\beta} \simeq X \otimes Y$ as $\left(\varrho^{K}, K\right)$-modules, where $\mathcal{G}^{K}$ acts on the second factor of $X \otimes Y$ and $K$ acts on the first factor (see [11, Lemma 5.3]). Similarly, $V_{\beta}^{\gamma, \lambda} \simeq X \otimes \operatorname{Hom}_{K}\left(X, V^{\gamma, \lambda}\right)$ as $\left(\mathcal{G}^{K}, K\right)$ modules. Thus $V_{\beta}$ is equivalent to an irreducible subquotient of the $\left(\mathcal{G}^{K}, K\right)$ module $V_{\beta}^{\gamma, \lambda}$. Let $Z_{1}$ and $Z_{2}$ be $\left(g^{K}, K\right)$-submodules of $V_{\beta}^{\gamma, \lambda}$ such that $Z_{1}$ $\subset Z_{2}$ and $Z_{2} / Z_{1}$ is equivalent to $V_{\beta}$. 
Define the subalgebra $A^{\beta}$ of $\mathcal{G}$ by $A^{\beta}=\left\{\left.x \in \mathcal{G}\right|^{\beta} x \subset \mathcal{G}^{\beta}\right\}$. Then $A^{\beta}=$ $K \varrho^{K}+\varrho^{\beta}$, by [11, Theorem 5.4]. Hence $Z_{1}$ and $Z_{2}$ are $A^{\beta}$-submodules of the $A^{\beta}$-module $V_{\beta}^{\gamma, \lambda}$, and $Z_{2} / Z_{1}$ is $A^{\beta}$-irreducible and is equivalent to the $A^{\beta}$. module $V_{\beta}$. Let $Z_{2}^{\text {min }}$ be the $\oint_{\text {-submodule of }} V^{\gamma, \lambda}$ generated by $Z_{2}$, and let

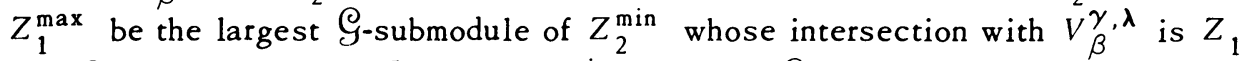
(see [11, Proposition 4.6]). Then $Z_{2}^{\min } / Z_{1}^{\max }$ is $\varrho_{\text {-irreducible, and }}$ $\left(Z_{2}^{\min } / Z_{1}^{\max }\right)_{\beta} \simeq Z_{2} / Z_{1} \simeq V_{\beta}$ as $A^{\beta}$-modules. Hence $Z_{2}^{\mathrm{min}} / Z_{1}^{\mathrm{max}} \simeq V$ as $\mathcal{G}_{\text {- }}$ modules, and thus as $(\varrho, K)$-modules, by [11, Theorem 4.9]. This proves the first assertion of the theorem, and hence the second. The last assertion follows from Lemma 2.2 and the last assertion of Theorem 8.7. Q.E.D.

The last assertion of Theorem 8.8 can easily be proved directly, as follows:

Proposition 8.9. Let $\gamma \in \hat{M}$ and $\lambda \in a^{\prime}$, and let $V$ be a (not necessarily irreducible) compatible (乌, M)-module which admits an effective bighest weight map with respect to $\gamma$ and $\lambda$. Then $\mathcal{Z}$ acts as scalars on $V$, inducing a bomo. morphism $\chi: \mathcal{Z} \rightarrow \mathrm{C}$, and $\chi=\chi_{\gamma, \lambda}^{\mathfrak{g}} \mid \mathcal{Z}$. In particular, Z acts on the $\mathrm{G}_{\text {-module }}$ $V^{\gamma, \lambda}$, and bence on any of its subquotients, according to the scalar bomomorphism $X_{\gamma, i \lambda+\rho}^{g} \mid \mathcal{Z}$.

Proof. Let $Z \in \gamma$, and let $f: V \rightarrow Z$ be the given effective highest weight map. For all $v \in V$ and $x \in \mathcal{G}^{M A}$, we have (recalling that $P_{\lambda}(x) \in \mathbb{M}^{M}$ by Lemma 8.2)

$$
f(x \cdot v)=P_{\boldsymbol{\lambda}}(x) \cdot f(v)-\left({ }_{\mu_{\mu}} \circ \chi^{\mathrm{m}} \circ P_{\boldsymbol{\lambda}}\right)(x) f(v)=\chi_{\gamma, \boldsymbol{\lambda}}^{\mathrm{g}}(x) f(v),
$$

by Lemma 8.2. In particular, if $x \in \mathcal{Z}$, then for all $y \in \varrho$ we have

$$
f(y \cdot(x \cdot v))=f(x \cdot(y \cdot v))=\chi_{\gamma, \boldsymbol{\lambda}}^{\mathbf{g}}(x) f(y \cdot v)=f\left(y \cdot \chi_{\gamma, \boldsymbol{\lambda}}^{\mathbf{g}}(x) v\right),
$$

so that $x \cdot v=\chi_{y, \lambda}^{\mathfrak{g}}(x) v$, by the definition of effectiveness. The last assertion of the theorem follows from Theorem 7.2. Q.E.D.

Two continuous Banach space representations of $G$ whose $\hat{K}$-multiplicities are finite are said to be infinitesimally equivalent if their (乌, $K$ )-modules of $K$-finite vectors are equivalent.

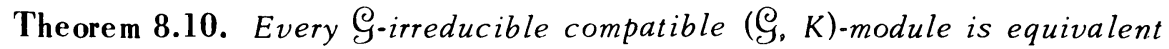
to the $\left(\biguplus_{,} K\right)$-module of $K$-finite vectors of some continuous topologically irreducible (not necessarily unitary) representation of $G$ on a Hilbert space, and this representation may be chosen to be a subquotient of a nonunitary principal series representation $\pi^{\gamma, \lambda}$ for some $\gamma \in \hat{M}$ and $\lambda \in \hat{A}$. Furthermore, every continuous topologically irreducible Banach space representation $\pi$ of $G$ such that the multiplicity of any element of $\hat{K}$ is finite is infinitesimally equivalent to an irreducible subquotient of some nonunitary principal series representation 
$\pi^{\gamma, \lambda}$. In particular, the multiplicity of any $\beta \in \hat{K}$ in $\pi$ is equal to or less than $m(\beta, \gamma) \leq d(\beta)$ (cf. Theorem 5.5). Moreover, $\mathcal{Z}$ acts on the space of K-finite vectors of $\pi$ according to the scalar bomomorphism $\chi_{\gamma, i \lambda+\rho}^{8} \mid \mathcal{Z}$.

Proof. The theorem follows easily from Theorem 8.8 and Harish-Chandra's results $[6(b)]$ relating Banach space representations of $G$ to the corresponding representations of $\mathcal{Y}$ on the spaces of $K$-finite vectors. (We note that Theorem 5 , p. 228 of $[6(b)]$ can be sharpened to the following statement, in our context: Suppose that $\pi$ is a continuous representation of $G$ on a Banach space $H$, with finite $\hat{K}$-multiplicities. Then there is a natural bijection between the set of closed $\pi(G)$-invariant subspaces $X$ of $H$ and the set of $\varrho_{\text {-submodules }} Y$ of the $\varrho_{\text {-module }}$ $V$ of $K$-finite vectors of $H$; the correspondence is given by $X \mapsto X \cap V, Y \mapsto$ closure (Y).) Q.E.D.

9. Equivalence under the Weyl group; finiteness theorems. Let $M^{\prime}$ be the normalizer of $A$ in $K$, so that the Weyl group $W=M^{\prime} / M$ is a finite group. Now $W$ acts in a natural way on $A$ and on $a$, and by contragredience, on $a^{\prime}$ and hence on $\hat{A}$. $W$ also acts in a natural way on $\hat{M}$, as follows: Let $\gamma \in \hat{M}$, and let $Z \in \gamma$, with $\pi: M \rightarrow$ Aut $Z$ the corresponding representation. For all $s \in W$, choose a representative $m_{s} \in M^{\prime}$. We define a new $M$-module structure on $Z$ by $m \cdot z=$ $\pi\left(m_{s}^{-1} m m_{s}\right) z$ for all $m \in M$ and $z \in Z$. The equivalence class of the resulting irreducible $M$-module depends only on $s$ and $\gamma$, and is denoted by $s \gamma$. The map from $W \times \hat{M}$ to $\hat{M}$ given by $(s, y) \mapsto s \gamma$ is indeed an action of $W$ on $\hat{M}$.

In $\$ 9$, we shall relate the notions of composition series (see the Appendix for relevant definitions), spherical function, infinitesimal spherical function and distribution character, and in the case of the nonunitary principal series, we shall relate these notions to $W$. We shall also prove the equivalence of various finiteness assumptions on $\mathcal{G}$-modules on which the center $\mathscr{Z}$ of $\mathcal{G}$ acts as scalars.

A continuous representation $\pi$ of $G$ on a separable Hilbert space $H$ is said to be admissible (cf. $[15, \$ 2])$ if

(1) as a $K$-representation $\pi$ is unitary,

(2) there is a constant $C$ such that for all $\beta \in \hat{K}$, the multiplicity of $\beta$ in $H$ is equal to or less than $C d(\beta)$, where as usual $d(\beta)$ denotes the dimension of any module in $\beta$.

Let $\pi$ be an admissible representation of $G$ on $H$. Let $C_{0}^{\infty}(G)$ denote the space of complex-valued $C^{\infty}$ functions of compact support on $G$. For all $f \in$ $C_{0}^{\infty}(G)$, the operator

$$
\pi(f)=\int_{G} f(g) \pi(g) d g
$$


(where $d g$ denotes Haar measure on $G$ ) is of trace class (see $[6(d)]$ ). The trace of this operator is denoted $T_{\pi}(f)$, and the resulting linear functional $T_{\pi}$ on $C_{0}^{\infty}(G)$ is a distribution, called the character of $\pi$ (see $[6(d)]$ ).

For every $\beta \in \hat{K}$, let $E_{\beta}^{\pi}: H \rightarrow H_{\beta}$ be the orthogonal projection onto the $\beta$ primary subspace $H_{\beta}$ of $H$. The spherical function $\phi_{\beta}^{\pi}: G \rightarrow \mathrm{C}$ associated with $\pi$ and $\beta$ is defined by

$$
\phi_{\beta}^{\pi}(g)=\operatorname{tr} E_{\beta}^{\pi} \pi(g) E_{\beta}^{\pi}
$$

for all $g \in G$. Then $\phi_{\beta}^{\pi}$ is an analytic function on $G$ (see [5] and [6(d)]).

Let $V^{\pi}$ denote the $K$-submodule of $K$-finite vectors of $H$. Then $V^{\pi}$ is naturally a $g_{R}$-module, and hence a compatible $(\varrho, K)$-module, under the action $\pi_{*}$ of $\mathcal{G}$ determined by

$$
\pi_{*}(x) v=\left.(d / d t) \pi(\exp t x) v\right|_{t=0}
$$

for all $x \in g_{\mathrm{R}}$ and $v \in V^{\pi}$ (see [6(b)]). For all $x \in \mathcal{G}$, let $D_{x}$ denote the left invariant differential operator on $G$ associated with $x$. Then for all $\beta \in \hat{K}$,

$$
\left(D_{x} \phi_{\beta}^{\pi}\right)(e)=\operatorname{tr} E_{\beta}^{\pi} \pi_{*}(x) E_{\beta}^{\pi},
$$

where $e$ denotes the identity element of $G$ (cf. [6(d), p. 235]). The infinitesimal spherical function associated with $\pi$ and $\beta$ is defined to be the complex-valued linear function $\chi\left(\eta\left(\left[V^{\pi}\right], \beta\right)\right)$ on $\mathcal{S}^{K}$.

We now establish three general facts about admissible representations.

Proposition 9.1. Let $\pi$ and $\sigma$ be admissible representations of $G$, and let $\beta \in \hat{K}$. Then the following conditions are equivalent:

(1) The spherical functions $\phi_{\beta}^{\pi}$ and $\phi_{\beta}^{\sigma}$ are equal.

(2) The infinitesimal spherical functions $\chi\left(\eta\left(\left[V^{\pi}\right], \beta\right)\right)$ and $\chi\left(\eta\left(\left[V^{\sigma}\right], \beta\right)\right)$ are equal.

(3) The $\mathrm{G}^{K}$-modules in the classes $\eta\left(\left[V^{\pi}\right], \beta\right)$ and $\eta\left(\left[V^{\sigma}\right], \beta\right)$ bave equivalent composition series.

(4) The K乌 $\mathrm{K}^{K}$-modules $V_{\beta}^{\pi}$ and $V_{\beta}^{\sigma}$ bave equivalent composition series.

Proof. We assert that the right-hand side of $(*)$ above is determined by its values for $x \in \mathfrak{K}^{K}$ only. Indeed, regard $\hat{K}$ as a subset of $\hat{K}$ (the set of equivalence classes of finite-dimensional irreducible $\mathcal{K}$-modules) in the natural way, and for every $\gamma \in \hat{\mathcal{K}}$, let $A^{\gamma, \beta}$ denote the subset of $\mathcal{G}$ which takes $V_{\beta}$ into

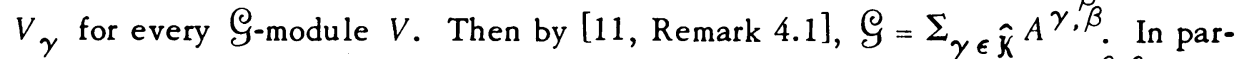
ticular, the right-hand side of $(*)$ is determined by its values for $x \in A^{\beta, \beta}$. But by $\left[11\right.$, Theorem 5.4], $A^{\beta, \beta}=\mathcal{K} \varrho^{K}+\varrho^{\beta}$, and this proves the assertion.

Since spherical functions are analytic, we thus have that $\phi_{\beta}^{\pi}=\phi_{\beta}^{\sigma}$ if and 
only if the $K \varrho^{K}$-modules $V_{\beta}^{\pi}$ and $V_{\beta}^{\sigma}$ have the same character. But two finitedimensional representations of an algebra over $\mathbf{C}$ have equal characters if and only if they have equivalent composition series. This shows that (1) and (4) are equivalent, and also that (2) and (3) are equivalent.

Let $X$ be a $\mathcal{K}$-module in the class $\beta$. Then

$$
V_{\beta}^{\pi} \simeq X \otimes \operatorname{Hom}_{K}\left(X, V^{\pi}\right)
$$

as $K / g^{\beta} \otimes \varrho^{K}$-modules, where $K / g^{\beta}$ acts on the first factor on the right, and $\mathcal{G}^{K}$ acts on the second (see [11, Lemma 5.3]). The same assertion also holds for $\sigma$ in place of $\pi$. This shows that (3) and (4) are equivalent. Q.E.D.

Proposition 9.2. Let $\pi$ and $\sigma$ be admissible representations of G. Then the following are equivalent:

(1) $T_{\pi}=T_{\sigma}$.

(2) The conditions of Proposition 9.1 bold for all $\beta \in \hat{K}$.

Proof. The equivalence of (1) and condition (1) of Proposition 9.1 for all $\beta \in \hat{K}$ follows from [6(d), \$7]; see also [15, Lemma 2.1]. Q.E.D.

Theorem 9.3. Let $\pi$ and $\sigma$ be admissible representations of $G$ and suppose that $T_{\pi}=T_{\sigma}$. Then if the $\mathrm{G}_{\text {-module }} V^{\pi}$ has a composition series, the same is true of the $\oint_{\text {-module }} V^{\sigma}$, and the two $\oint_{\text {-modules bave equivalent composition }}$

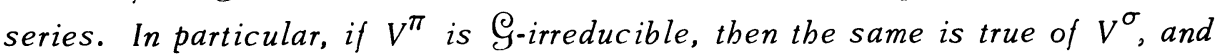
the two -modules are equivalent.

Proof. By Propositions 9.1 and 9.2, the $K \mathrm{~K}^{K}$-modules $V_{\beta}^{\pi}$ and $V_{\beta}^{\sigma}$ have equivalent composition series. The theorem now follows immediately from Theorem 11.1 and Remark 11.2 in the Appendix. Q.E.D.

Remark 9.4. The last assertion of Theorem 9.3 was obtained by N. Wallach [15, Theorem 2.1]. Also, Corollary 11.10(ii) in the Appendix and Proposition 9.1 immediately imply [15, Corollary 2.1$]$, a related result.

Remark 9.5. Our proof of Theorem 9.3 is very simple in two special cases-

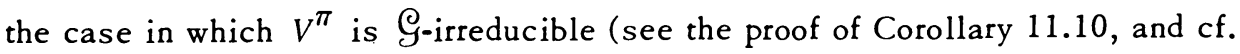
Remark 9.4) and the case in which $V^{\sigma}$ is assumed to have a composition series for $९$ (see Remark 11.11).

We now apply the above results to the study of the nonunitary principal series. We note that for all $\gamma \in \hat{M}$ and $\lambda \in \hat{A}, \pi^{\gamma, \lambda}$ is an admissible representation of $G$. Let $T_{\gamma, \lambda}$ denote the character of $\pi^{\gamma, \lambda}$.

The following theorem is essentially due to Harish-Chandra (see [6(e)] and [15, Theorem 3.1]):

Theorem 9.6. For all $\gamma \in \hat{M}, \lambda \in \hat{A}$ and $s \in W, T_{\gamma, \lambda}=T_{s \gamma, s \lambda}$. 
We could now combine Theorem 9.6 with Propositions 9.1 and 9.2 and Theorem 9.3. But we can be more specific, in view of the following result which we prove below, following several people's suggestions, using Harish-Chandra's deep result Theorem 9.14:

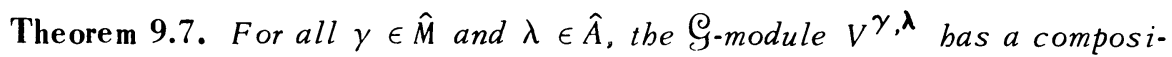
tion series.

For all $\gamma \in \hat{M}, \lambda \in \hat{A}$ and $\beta \in \hat{K}$, let $\phi_{\beta}^{\gamma}, \lambda=\phi_{\beta}^{\pi^{\gamma}, \lambda}$. We now have

Theorem 9.8. Let $\gamma \in \hat{M}, \lambda \in \hat{A}$ and $s \in W$. Then

(1) the 乌-modules $V^{\gamma, \lambda}$ and $V^{s \gamma, s \lambda}$ bave equivalent composition series,

(2) $p_{\beta, \gamma i \lambda_{+} \rho}\left|\varrho^{K}=p_{\beta, s \gamma, i s \lambda+\rho}\right| \varrho^{K}$ for all $\beta \in \hat{K}$ such that $m(\beta, \gamma)>0$,

(3) $T_{\gamma, \lambda}=T_{s \gamma, s \lambda}$,

(4) $\phi_{\beta}^{\gamma, \lambda}=\phi_{\beta}^{s \gamma, s, \lambda}$ for all $\beta \in \hat{K}$,

(5) $\chi\left(\eta\left(\left[V^{\gamma, \lambda}\right], \beta\right)\right)=\chi\left(\eta\left(\left[V^{s \gamma, \bar{s} \lambda}\right], \beta\right)\right)$ for all $\beta \in \hat{K}$,

(6) the $\mathrm{G}^{K}$-modules in the classes $\eta\left(\left[V^{\gamma, \lambda}\right], \beta\right)$ and $\eta\left(\left[V^{s \gamma}, s \lambda\right], \beta\right)$ bave equivalent composition series for all $\beta \in \hat{K}$,

(7) the K乌 $\mathcal{K}^{K}$-modules $V_{\beta}^{\gamma, \lambda}$ and $V_{\beta}^{s \gamma, s \lambda}$ bave equivalent composition series for all $\beta \in \hat{K}$.

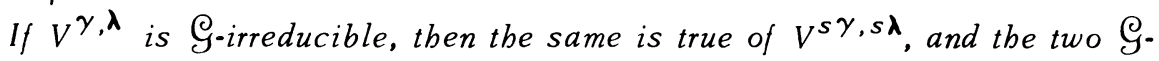
modules are equivalent. In this case, $V^{\gamma, \lambda}$ admits an effective bighest weight map with respect to $s \gamma$ and is $\lambda+\rho$.

Proof. All the assertions are clear from the above results in this section and Theorems 7.2 and 7.3. Q.E.D.

Remark 9.9. It would be interesting to determine all the highest weight maps for the modules $V^{\gamma, \lambda}$; cf. [7(b), p. 125, Theorem 6.3] and [16] for special cases.

Remark 9.10. In [2, p. 193, Théorème 7;2], F. Bruhat states that in the unitary case (i.e., when $\lambda \in a^{\prime}$ is real, in our notation), the representations $\pi_{\gamma, \boldsymbol{\lambda}}$ and $\pi_{s \gamma, s \boldsymbol{\lambda}}(\gamma \in \hat{M}, s \in W)$ are equivalent. His proof is based on assertion (4) of Theorem 9.8, but his proof of this assertion has a gap, as R. Goodman has pointed out to us; specifically, formula $(7 ; 33)$ on p. 195 of [2] seems to be unjustified.

Remark 9.11. Theorem 9.8 can be proved without using Theorem 9.6, as follows: For all $x \in \mathcal{G}^{K}, \gamma \in \hat{M}, s \in W$ and $\beta \in \hat{K}$ such that $m(\beta, \gamma)>0$, $p_{\beta, \gamma, i \lambda_{+} \rho}(x)$ and $p_{\beta, s \gamma, i s \lambda+\rho}(x)$ are polynomial functions in $\lambda \in a^{\prime}$. Hence assertion (2) of Theorem 9.8 (and hence all of Theorem 9.8) will follow if any one of the assertions (1)-(7) is proved for a Zariski dense set of $\lambda \in a^{\prime}$ (assuming that $\gamma \in \hat{M}$ and $s \in W$ are fixed). In particular, it is sufficient to prove (1) for a Zariski dense set of $\lambda \in a^{\prime}$. Hence it is sufficient to find a Zariski 
dense subset $S$ of $a^{\prime}$ such that, for all $\lambda \in S, V^{\gamma, \lambda}$ is $\mathcal{S}_{\text {-irreducible and there }}$

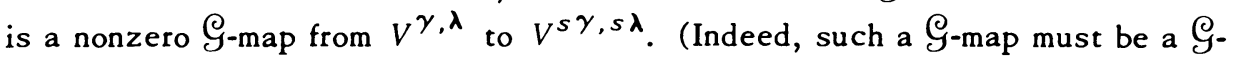
isomorphism.) To construct $S$, we can use the formal intertwining operators between $V^{\gamma, \lambda}$ and $V^{s \gamma, s \lambda}$ introduced by R. A. Kunze and E. M. Stein [10, p. 390, formula (1.5)]. These operators are well defined and nonzero as $\lambda$ ranges through a certain nonempty open subset of $a^{\prime}$, in view of the convergence theorem of $S$. G. Gindikin and F. I. Karpelevic [4, Theorem 1] (cf. also [7(b), p. 83]). We can now use the result of A. W. Knapp and Stein [8] and G. Schiffmann [14] that the formal intertwining operators can be meromorphically continued to give (finite) nonzero intertwining operators between $V^{\gamma, \lambda}$ and $V^{s \gamma, s \lambda}$ for most $\lambda \epsilon a^{\prime}$, including a Zariski dense set of real, regular $\lambda$. We may choose $S$ to be this set, in view of Bruhat's irreducibility result [2, p. 193, Théorème 7;2] for the regular members of the unitary principal series. Another subset $S$ may be constructed as follows: The irreducibility of $V^{\gamma, \lambda}$ for a single $\lambda \in a^{\prime}$ (which follows for example from Bruhat's irreducibility result) implies the irreducibility for a topologically dense set of $\lambda \in a^{\prime}$ (this was pointed out to us by B. Kostant and N. Wallach). This dense set must intersect the (open) domain of convergence of the formal intertwining operators in a Zariski dense set of $\lambda \in a^{\prime}$, and we may choose $S$ to be this intersection.

Remark 9.12. Let $\beta$ and $\gamma$ be the classes of the trivial one-dimensional representations of $K$ and $M$, respectively. In this case, Theorem 9.8(2) was obtained by Harish-Chandra, essentially by using his method of proof of Theorem 9.6 (see Remark 3.4, [6(g), p. 260, Theorem 1] and [7(a), p. 431, Lemma 6.14]). We can give a simpler proof, as follows: Let $s \in W$, and suppose that $\lambda \epsilon a^{\prime}$ is in the domain of convergence of the formal intertwining operator which takes $V^{\gamma, \lambda}$ to $V^{s \gamma, s \lambda}\left(=V^{\gamma, s \lambda}\right)$ (see Remark 9.11). It is easy to see that the set of such $\lambda$ such that the intertwining operator is nonzero on the one-dimensional space $V_{\beta}^{\gamma, \lambda}$ is precisely the subset of $a^{\prime}$ such that the integral $[7(b)$, p. 83 , formula (8)] defining the partial $c$-function associated with $s$ converges and is nonzero. Since this set is nonempty and open, and is thus Zariski dense, we obtain Theorem 9.8(5) for $\beta$ and $\gamma$ trivial and all $\lambda$ in a Zariski dense subset of $a^{\prime}$, and this is sufficient to prove Theorem 9.8(2) for $\beta$ and $\gamma$ trivial (see the beginning of Remark 9.11).

Remark 9.13. Theorem 9.8(2) may be regarded as a generalization of [12, Theorem 2.3 (2.56)] (cf. Remarks 3.5 and 7.5).

We now prove Theorem 9.7 and some related finiteness results. The proofs are based on the following deep result:

Theorem 9.14 (Harish-Chandra). Let $\chi: \mathcal{Z} \rightarrow \mathrm{C}$ be a bomomorphism (we recall that $\mathcal{Z}$ is the center of $\mathcal{G}$ ). Then there are only finitely many infinitesimally inequivalent (see \$8) continuous topologically irreducible Hilbert space repre- 
sentations of $G$ with finite $\hat{K}$-multiplicities such that $\mathcal{Z}$ acts on the $\mathcal{G}_{\text {-module of }}$ $K$-finite vectors according to $\chi$.

Proof. See the first part of the proof of Lemme 1 of [3(c)]. (The proof is based on Harish-Chandra's deep theorem [6(i)] that every distribution character is actually a locally summable function on G.) Q.E.D.

Corollary 9.15. Let $\chi: \mathcal{Z} \rightarrow \mathbf{C}$ be a bomomorphism. Then there are only

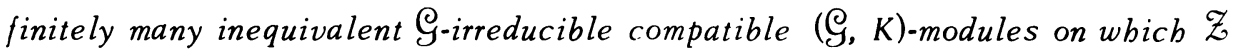
acts according to $\gamma$.

Proof. The corollary follows immediately from the theorem and the first assertion of Theorem 8.10. Q.E.D.

Theorem 9.7 follows from the fact that (1) implies (5) in the following theorem, in view of Proposition 8.9. However, we note that although Theorem 8.10 is used in the proof of this theorem, it is not required to prove Theorem 9.7.

Theorem 9.16.(4) Let $V$ be a compatible (乌, K)-module on which $\mathcal{Z}$ acts according to a scalar homomorphism. Then the following conditions are equivalent:

(1) For all $\beta \in \hat{K}, m([V], \beta)<\infty$.

(2) There is a constant $C$ such that, for all $\beta \in \hat{K}, m([V], \beta) \leq C d(\beta)$.

(3) $V$ is a finitely generated $\mathcal{G}_{\text {-module. }}$

(4) $V$ is a Noetberian -module.

(5) $V$ has a composition series as a -module.

Proof. We first show that (1) implies (5). Suppose that (1) holds. If $V$ does not have a composition series, then for any $n \geq 0$, we can find a sequence of $\mathcal{G}_{\text {- }}$ submodules

$$
V=V_{0} \supset V_{1} \supset \ldots \supset V_{n}=0
$$

such that $V_{i} \neq V_{i+1}$ for all $i=0, \cdots, n-1$. Now every $\varrho_{\text {-submodule of } V}$ is the direct sum of its intersections with the spaces $V_{\beta}(\beta \in \hat{K})$. Using this observation, it is easy to construct $\mathcal{G}_{\text {-submodules }} W_{i}$ and $X_{i}$ of $V_{i}$ such that

$$
V_{i} \supset W_{i} \supset X_{i} \supset V_{i+1}
$$

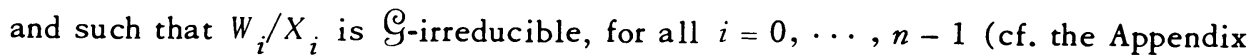
and [11, Proposition 4.6]). Now $\mathcal{Z}$ acts on each $W_{i} / X_{i}$ according to a fixed scalar homomorphism, and so by Corollary 9.15 there exist finitely many $\oint_{\text {- }}$ modules $Z_{1}, \ldots, Z_{p}$ such that each $W_{i} / X_{i}$ is equivalent to one of the $Z_{j}$. For each $j=1, \ldots, p$, choose $\beta_{j} \in \hat{K}$ such that $m\left(\left[Z_{j}\right], \beta_{j}\right)>0$, and let $m_{j}=$ $m\left([V], \beta_{j}\right)$, so that $m_{j}<\infty$ by hypothesis. Then there can only be at most $m_{j}$ indices $i$ between 0 and $n-1$ such that $W_{i} / X_{i} \simeq Z_{j}$. Thus $n$ cannot be larger than $m_{1}+\cdots+m_{p}$, a contradiction, and so (1) implies (5).

(4) Theorem 9.16 appears to establish, for groups with finite center, a conjecture of I. M. Gel' fand (see 2(c), footnote 5). 
Now it is standard that (5) implies (4) and that (4) implies (3). The fact that (3) implies (1) follows easily from [6(b), p. 195, Theorem 1]. Finally, (2) trivially implies (1), and (5) implies (2) in view of Theorem 5.4; we may choose $C$ to be the number of irreducible subquotients associated with a composition series of $V$. Q.E.D.

Remark 9.17. Corollary 9.15 and part of Theorem 9.16 are obtained in [3(c), Lemme 1 and Lemme 2] for the special case in which $G$ is a matrix group.

10. The image of the map $p_{\beta} \mid \varrho^{K}$. In this section, we shall compute the image of the map $p_{\beta} \mid \varrho^{K}(\beta \in \hat{K})$ and obtain related results. We also state two conjectures.

For all $s \in W$ (see $\$ 9$ ), let $s_{*}$ denote the affine transformation of $a^{\prime}$ given by $s_{*} \lambda=s(\lambda-\rho)+\rho$ for all $\lambda \in a^{\prime}$. Let $s^{*}$ denote the algebra automorphism of $\mathbb{Q}$ (identified with the algebra of polynomial functions on $a^{\prime}$ ) given by $\left(s^{*} x\right)(\lambda)=$ $x\left(s_{*}^{-1} \lambda\right)$ for all $x \in \mathbb{Q}, \lambda \in a^{\prime}$. This gives an action $W \rightarrow W^{*}=\left\{s^{*} \mid s \in W\right\}$ of $W$ as automorphisms of $\mathbb{Q}$. We define $\mathbb{Q}^{*}$ to be the set of $W$-invariants in $\mathbb{Q}$ under this action.

We recall from $\$ 3$ the definitions of the symbols $\Gamma_{\beta}$ and $p_{\beta, \Gamma}$, and we recall from $\S 4$ the map $\lambda: S(g) \rightarrow \mathcal{G} .(5)$ We denote by $S(p)^{K}$ the set of $K$-invariants in $S(p)$ under the natural action of $K$ on $S(p)$.

Theorem 10.1. Let $\beta \in \hat{K}$ and let $\Gamma$ be a nonempty $W$-stable subset of $\Gamma_{\beta}$. Then the image of the map $p_{\beta, \Gamma}: \varrho^{K} \rightarrow \mathbb{Q}$, and in particular, the map $p_{\beta}=p_{\beta, \Gamma_{\beta}}$ : $\Theta^{K} \rightarrow \mathbb{A}$, is precisely $\mathfrak{Q}^{*}$. Moreover, the restriction of $p_{\beta_{0} \Gamma}$ to $\lambda\left(S(p)^{K}\right)$ is $a$

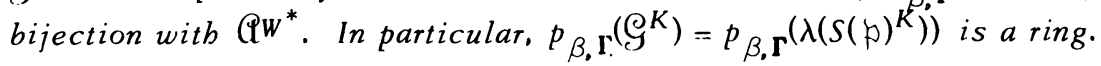

Proof. For all $s \in W, x \in \mathcal{C}^{K}$ and $\lambda \in a^{\prime}$, we have

$$
p_{\beta, \Gamma, \lambda}(x)=\sum_{\gamma \in \Gamma} d(\gamma) p_{\beta, \gamma, \lambda}(x)=\sum_{\gamma \in \Gamma} d(\gamma) p_{\beta, s \gamma, s_{*} \lambda}(x)
$$

(by Theorem 9.8(2))

$$
=\sum_{\gamma \in \Gamma} d(\gamma) p_{\beta_{0} \gamma, s_{*} \lambda^{\lambda}}(x)
$$

(since $\Gamma$ is $W$-stable)

$$
=p_{\beta, \Gamma, s_{*} \lambda}(x),
$$

so that

$$
s^{*} p_{\beta_{0} \Gamma} \Gamma^{(x)}=p_{\beta_{0} \Gamma}(x)
$$

Hence $p_{\beta_{0}}: \varrho^{K} \rightarrow \mathcal{Q W}^{*}$.

(5) The reader should note that the symbol $\lambda$ is used in two different ways in this section. 
Using the notation of Lemma 4.7, we note that $p_{\beta, \Gamma}$ is a map essentially of the form $P_{T}$; specifically, if we take $V=\mathbf{C}$ and $T: \mathcal{K} \rightarrow \mathbf{C}$ to be any linear map on $\mathcal{K}$ defined by

$$
T=\sum_{\gamma \in \Gamma} d(\gamma) \operatorname{tr}_{\beta, \gamma}{ }^{\circ} \pi_{\beta, \gamma}
$$

on $\mathcal{K}^{M}$ (see $\S 3$ ), then $P_{T}: \mathcal{G} \rightarrow S(a)$ is a linear map which agrees with the map $p_{\beta, \Gamma}: \mathfrak{G}^{M} \rightarrow \mathbb{Q}$ on $\mathcal{G}^{M}$. Since

$$
T(1)=\sum_{\gamma \in \Gamma} d(\gamma) m(\beta, \gamma) \neq 0,
$$

we have by Lemma 4.7 that $p_{\beta, \Gamma} \mid \lambda\left(S(p)^{K}\right)$ is injective.

To show that $p_{\beta, \Gamma}\left(\lambda\left(S(p)^{K}\right)\right)=\mathbb{Q} w^{*}$, we let $y \in \mathbb{Q} W^{*}$ and prove by induction on $d=\operatorname{deg} y$ that there exists $x \in S(p)^{K}$ such that $p_{\beta, \Gamma}(\lambda(x))=y$. The assertion is clearly true if $d=0$. Assume that $d>0$. We identify $\mathbb{A}$ with $S(a)$ via the isomorphism $\lambda$.

Let $S(a)^{W}$ denote the set of $W$-invariants in $S(a)$ under the action induced by the natural action of $W$ on $a$. Let $g: S(p) \rightarrow S(a)$ denote the projection map with respect to the decomposition $S(p)=S(a) \oplus q S(p)$ (see $\$ 4$ for the definition of $q$ ). Then by the theorem of C. Chevalley (see [7(a), p. 430, Theorem 6.10]), $g$ : $S(p)^{K} \rightarrow S(a)^{W}$ is an isomorphism. Moreover, $\operatorname{deg} z=\operatorname{deg} g(z)$ for all $z \in S(p)$, where deg denotes the ordinary degree function on $S(g)$.

Let $A_{\rho}: S(a) \rightarrow S(a)$ be the algebra automorphism determined by the condition $A_{\rho}(u)=u+\rho(u)$ for all $u \in a$. Then for all $v \in S(a)$, the highest degree terms of $v$ and $A_{\rho}(v)$ coincide.

Now $A_{\rho}(y) \in S(a)^{W}$, so that by Chevalley's theorem, there exists $z \in S(. p)^{K}$ such that $g(z)=A \rho(y)$. In the notation of Lemma 4.7, with $T$ as above, we have $f_{T}(\lambda(z))=c g(z)$, where $c$ is a nonzero constant (specifically, $c=T(1)$ ). Hence by Lemma 4.7 , we have that

$$
\operatorname{deg}\left(p_{\beta, \Gamma}(\lambda(z))-c g(z)\right)<\operatorname{deg} \lambda(z)=\operatorname{deg} z
$$

(since $z \in S(p)$ )

$$
=\operatorname{deg} g(z)=\operatorname{deg} A_{\rho}(y)=d .
$$

Moreover, $y=A_{\rho}^{-1}(g(z))$ has the same $d$ th degree term as $g(z)$. Thus

$$
\operatorname{deg}\left(p_{\beta, \Gamma}(\lambda(z))-c y\right)<d,
$$

and

$$
p_{\beta, \Gamma}(\lambda(z))-c y \in \mathfrak{Q} W^{*},
$$

by the first part of the theorem. Hence by the inductive assumption, there exists $w \in S(p)^{K}$ such that $p_{\beta, \Gamma}(\lambda(w))=p_{\beta, \Gamma}(\lambda(z))-c y$. Choosing $x=c^{-1}(z-w)$, we 
have that $x \in S(p)^{K}$ and that $p_{\beta, \Gamma}(\lambda(x))=y$, completing the induction. Q.E.D.

Remark 10.2. Choosing $\beta$ to be the trivial class in Theorem 10.1, we have Harish-Chandra's theorem (see [ $6(\mathrm{~g})$, p. 260, Theorem 1 and p. 262, Lemma 19] and [7(a) , p. 431, Lemma 6.14]) that the image of the classical map $p_{\beta} \mid \oint^{K}$ (see Remark 3.4) is precisely $\mathscr{Q} W^{*}$, and that $p_{\beta}: \lambda\left(S(p)^{K}\right) \rightarrow \mathbb{Q} W^{*}$ is a linear isomorphism. See also Remark 9.12.

Conjecture 10.3. For every $\beta \in \hat{K}$, we conjecture that the image

$$
P_{\beta}\left(\mathrm{g}^{K}\right) \subset \mathbb{Q} \otimes\left(K^{M} / K^{M} \cap g^{\beta}\right)
$$

is $W$-invariant, where $W$ acts on $K^{M} / K^{M} \cap g^{\beta}$ via the quotient of the natural action of $W$ on $K^{M}$, and where $W$ acts on $\mathcal{Q}$ via $W^{*}$. This would define a natural action of $W$ on $\mathcal{S}^{K} / \mathscr{g}^{K} \cap \mathcal{G}^{\beta}$.

Conjecture 10.4. For all $\beta \in \hat{K}, \gamma, \gamma_{1} \in \hat{M}$ and $\lambda, \lambda_{1} \in \hat{A}$ such that $m(\beta, \gamma)$ $>0$ and $m\left(\beta, \gamma_{1}\right)>0$, we conjecture that $p_{\beta, \gamma, \lambda}\left|\biguplus^{K}=p_{\beta, \gamma_{1}, \lambda_{1}}\right| \varrho^{K}$ only if there exists $s \in W$ such that $\gamma_{1}=s \gamma$ and $\lambda_{1}=s_{*} \lambda$, and that the image $p_{\beta, \gamma}\left(\varrho^{K}\right) \subset \mathbb{Q}$ is precisely the subset of $\mathbb{Q}$ left fixed by $W_{\gamma}^{*}$, where $W_{\gamma}^{*}=\left\{s^{*} \mid s \in W\right.$ and $s$ fixes $\gamma\}$ (cf. [12, \$3.6] and Remarks 3.5 and 9.13). This would give information on all the possible equivalences among composition quotients of the $\oint_{\text {-modules }}$ $\mathrm{V}^{\gamma, \lambda}$. Note that Harish-Chandra's converse of Theorem 9.6 above (see [6(e)] and [15, Theorem 3.1]) does not appear sufficient to prove even the first assertion of this conjecture.

11. Appendix: Equivalence of composition series for certain modules. In this Appendix, we establish a general module-theoretic result (Theorem 11.1), which together with [6(c), p. 36, Corollary 2] or, alternatively, the results of [11] (see Remark 11.2) is used to prove Theorem 9.3 above.

Let $\mathcal{B}$ be a ring (with 1 ), and $V$ a (left) $B$-module. A composition series of $V$ is a finite sequence of $\mathfrak{B}$-submodules

$$
V=V_{0} \supset V_{1} \supset \cdots \supset V_{n}=0
$$

such that $V_{i} / V_{i+1}$ is an irreducible $B$-module for all $i=0, \cdots, n-1$. If

$$
W=W_{0} \supset W_{1} \supset \ldots \supset W_{m}=0
$$

is a composition series for a $\mathbb{B}$-module $W$, then the two composition series are equivalent if $m=n$ and the factor modules $V_{i} / V_{i+1}(0 \leq i \leq n-1)$ are equiva lent to the factor modules $W_{i} / W_{i+1}(0 \leq i \leq m-1)$, up to order. By the JordanHölder theorem, any two composition series of a $B$-module are equivalent. Moreover, if a $\mathfrak{B}$-module $V$ has a composition series, then any strictly descending chain

$$
X_{1} \supset X_{2} \supset \cdots \supset X_{p}
$$


of $\Re$-submodules of $V$ can be extended to a composition series.

Now let $\mathfrak{Q}$ be a subring of $\mathfrak{B}$, and regard $\mathfrak{B}$-modules as $\mathfrak{Q}$-modules by restriction. Let $\Gamma$ be a set of equivalence classes of irreducible $\mathbb{Q}$-modules. For every $\mathfrak{Q}$ module $V$ and $\gamma \in \Gamma$, let $V_{\gamma}$ denote the $\gamma$-primary submodule of $V$. Suppose that for each $\gamma \in \Gamma$, there is a subring $A^{\gamma}$ of $\mathbb{B}$ with the following properties:

(1) For every $B$-module $V, V_{\gamma}$ is stable under $A^{\gamma}$.

(2) For every irreducible $B$-module $V$ such that $V_{\gamma} \neq 0, V_{\gamma}$ is irreducible under $A^{\gamma}$.

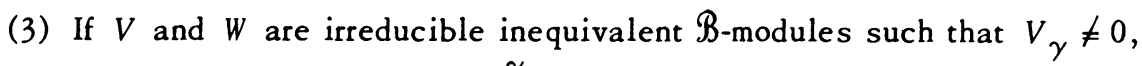
then $V_{\gamma}$ and $W_{\gamma}$ are inequivalent $A^{\gamma}$-modules.

Then we have the following result, which is proved below:

Theorem 11.1. Let $V^{1}$ be a $\mathfrak{B}$-module which has a composition series. Then for all $\gamma \in \Gamma$, the $A^{\gamma}$-module $V_{\gamma}^{1}$ bas a composition series. Suppose that $V^{2}$ is a B-module, that $V^{i}=\Sigma_{\gamma \in \Gamma} V_{\gamma}^{i}(i=1,2)$, and that for every $\gamma \in \Gamma$, the $A^{\gamma}$. modules $V_{\gamma}^{1}$ and $V_{\gamma}^{2}$ bave equivalent composition series. Then $V^{2}$ bas a composition series, and $V^{1}$ and $V^{2}$ bave equivalent composition series.

Remark 11.2. In the notation and with the assumptions of $[11, \S 4]$ (for all $a \in \hat{\mathbb{Q}}$ ), Theorem 11.1 holds for $\Gamma=\hat{\mathbb{Q}}$ and $A^{\gamma}=A^{\gamma, \gamma}$ for all $\gamma \in \hat{\mathbb{Q}}$, in view of [11, Propositions 3.3 and 4.5, and Theorem 4.9]. In the notation and with the assumptions of $[11, \S 5]$ (for all $\alpha \in \hat{\mathfrak{Q}}$ ), Theorem 11.1 holds for $\Gamma=\hat{\mathbb{Q}}$ and $A^{\gamma}=$ $\mathfrak{A B}^{\mathfrak{a}}$ for all $\gamma \in \hat{\mathbb{Q}}$, in view of [11, Theorem 5.5]. In particular, Theorem 11.1 holds for $\mathfrak{B}=\mathscr{G}, \mathbb{A}=\mathcal{K}, \Gamma=\hat{K}$ and $A^{\gamma}=\mathcal{K} \mathcal{G}^{K}$ for all $\gamma \in \hat{K}$. (The fact that assumptions (1), (2) and (3) hold in this case also follows from [6(c), p. 36, Corollary 2].)

To prove Theorem 11.1, we establish the following lemmas:

Lemma 11.3. Let $V$ be a B-module which has a composition series. Then for all $\gamma \in \Gamma$, the $A^{\gamma}$-module $V_{\gamma}$ bas a composition series.

Proof. Let

$$
V=V_{0} \supset V_{1} \supset \cdots \supset V_{n}=0
$$

be a composition series of $V$. Then by assumption (2), the $A^{\gamma}$-module $\left(V_{i} / V_{i+1}\right)_{\gamma}$ is either zero or irreducible $(0 \leq i \leq n-1)$. But the $A^{\gamma}$-module $\left(V_{i}\right)_{\gamma} /\left(V_{i+1}\right)_{\gamma}$ naturally injects into $\left(V_{i} / V_{i+1}\right)_{\gamma}$. Hence if $\left(V_{i}\right)_{\gamma} \neq\left(V_{i+1}\right)_{\gamma}$, then $\left(V_{i}\right)_{\gamma} /\left(V_{i+1}\right)_{\gamma}$ is $A^{\gamma}$-irreducible, and so the distinct members of the chain

$$
V_{\gamma}=\left(V_{0}\right)_{\gamma} \supset\left(V_{1}\right)_{\gamma} \supset \cdots \supset\left(V_{n}\right)_{\chi}=0
$$

form a composition series of the $A^{\gamma}$-module $V_{\gamma}$. Q.E.D.

Lemma 11.4. Let $V$ be a B-module such that $V=\Sigma_{\gamma \in \Gamma} V_{\gamma}$. Let $\gamma \in \Gamma$, and 
suppose that the $A^{\gamma}$-module $V_{\gamma}$ is irreducible. Then there exist $B$-submodules $X$ and $Y$ of $V$ such that $X \subset Y, V_{\gamma} \subset Y, X \cap V_{\gamma}=0$, and $Y / X$ is B-irreducible.

Proof. Let $Y$ be the $\mathfrak{B}$-submodule of $V$ generated by $V_{\gamma}$, and let $X$ be the sum of the $B$-submodules of $Y$ which intersect $V_{\gamma}$ in 0 . Then $X \cap V_{\gamma}=0$, since every $\mathbb{Q}$-submodule of $V$, and in particular, every $\Re$-submodule of $V$, is the direct sum of its intersections with the primary $\mathbb{Q}$-submodules of $V$. It is clear that $Y / X$ is $B$-irreducible. Q.E.D.

Remark 11.5. The construction of the irreducible $B$-module $Y / X$ in Lemma 11.4 generalizes the construction of the irreducible representations $\hat{\pi}_{\lambda, \nu}$ in $[12$, p. 397].

Lemma 11.6. Let $V$ be a B-module such that $V=\Sigma_{\gamma \in \Gamma} V_{\gamma}$. Let $\gamma \in \Gamma$, and suppose that $V_{\gamma} \neq 0$ and that $V_{\gamma}$ is not $A^{\gamma}$-irreducible. Then there is a B-submodule $W$ of $V$ such that $0 \neq W \cap V_{\gamma} \neq V_{\gamma}$.

Proof. Define $X$ and $Y$ exactly as in the proof of Lemma 11.4, so that in particular, $X \cap V_{\gamma}=0$. Now $(Y / X)_{\gamma}$ and $V_{\gamma}$ are equivalent as $A^{\gamma}$-modules, so that $(Y / X)_{y}$ is not $A^{\gamma}$-irreducible. Hence $Y / X$ is not $B$-irreducible, by assumption (2). Thus there is a $B$-submodule $W$ of $V$ such that $X \subset W \subset Y$ and such that $0 \neq W \cap V_{\gamma} \neq V_{\gamma}$. Q.E.D.

Remark 11.7. In the situation of $[11, \$ 4]$ (see Remark 11.2), Lemma 11.6 follows immediately from the existence of extensions [11, Proposition 4.6].

Lemma 11.8. Let $V$ be a $B$-module such that $V=\Sigma_{\gamma \in \Gamma} V_{\gamma}$. Let $\gamma \in \Gamma$, and suppose that the $A^{\gamma}$-module $V_{\gamma}$ has a composition series. Let

$$
V=X_{0} \supset Y_{1} \supset X_{1} \supset Y_{2} \supset X_{2} \supset \cdots \supset Y_{n} \supset X_{n} \supset Y_{n+1}=0
$$

be a chain of $\mathfrak{B}$-submodules of $V$. Choose $i(0 \leq i \leq n)$ such that

$$
X_{i} \cap V_{y} \neq Y_{i+1} \cap V_{\gamma} \text {. }
$$

Then there exists a chain of $\mathfrak{B}$-submodules

$$
X_{i}=X_{i 0} \supset Y_{i 1} \supset X_{i 1} \supset Y_{i 2} \supset X_{i 2} \supset \ldots \supset Y_{i m_{i}} \supset X_{i m_{i}} \supset Y_{i, m_{i}+1}=Y_{i+1}
$$

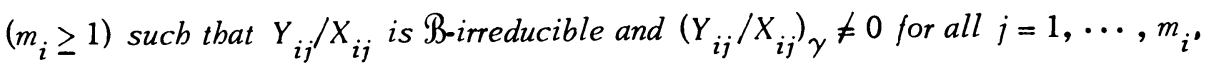
and such that

$$
X_{i j} \cap V_{\gamma}=Y_{i, j+1} \cap V_{\gamma}
$$

for all $j=0, \ldots, m_{i}$

Proof. If $\left(X_{i} \cap V_{\gamma}\right) /\left(Y_{i+1} \cap V_{\gamma}\right)$ is not $A^{\gamma}$-irreducible, then applying Lemma

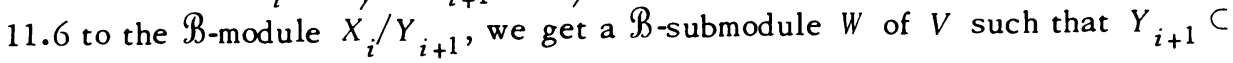
$W \subset X_{i}$ and such that 


$$
Y_{i+1} \cap V_{\gamma} \neq W \cap V_{y} \neq X_{i} \cap V_{\gamma} \text {. }
$$

Since the $A^{\gamma}$-module $V_{\gamma}$ has a composition series, we can repeat this argument to obtain a finite chain of $\mathscr{B}$-submodules between $X_{i}$ and $Y_{i+1}$ whose $\gamma$-primary $(\mathcal{A}$-submodules form a strictly decreasing chain whose successive quotients are $A^{\gamma}$-irreducible. Applying Lemma 11.4 to the successive quotients of $B$-modules, we get the desired chain. Q.E.D.

Proof of Theorem 11.1. The first assertion is proved in Lemma 11.3.

Let $Q_{1}, \ldots, Q_{n}$ be the irreducible $B$-modules which are the composition quotients of $V^{1}$, repeated according to multiplicity. Choose $\gamma_{1}, \ldots, \gamma_{s} \in \Gamma$ such that for all $i=1, \ldots, n$, there exists $j=1, \ldots, s$ such that $\left(Q_{i}\right)_{\gamma_{j}} \neq 0$.

We first apply Lemma 11.8 to $V=V^{2}, \gamma=\gamma_{1}$, and the trivial chain $V^{2}=$ $X_{0} \supset Y_{1}=0$. The result is a chain of $\mathfrak{B}$-modules

$$
V^{2}=X_{0}=X_{00} \supset Y_{01} \supset X_{01} \supset \cdots \supset Y_{0 m_{0}} \supset X_{0 m_{0}} \supset Y_{0, m_{0}+1}=Y_{1}=0
$$

such that $Y_{0 j} / X_{0 j}$ is $\mathcal{B}$-irreducible, each $\left(Y_{0 j} / X_{0 j}\right)_{\gamma_{1}} \neq 0$, and each

$$
X_{0 j} \cap V_{\gamma_{1}}=Y_{0, j+1} \cap V_{\gamma_{1}} \text {. }
$$

Now we apply Lemma 11.8 to $V=V^{2}, \gamma=\gamma_{2}$, and the above chain. We continue successively to apply Lemma 11.8 to $\gamma_{j}$ and the chain resulting from $\gamma_{j-1}$ until we reach $\gamma_{s}$. The final result is a chain of $\mathfrak{B}$ modules

$$
V^{2}=U_{0} \supset w_{1} \supset U_{1} \supset w_{2} \supset U_{2} \supset \ldots \supset w_{t} \supset U_{t} \supset w_{t+1}=0
$$

such that $W_{i} / U_{i}(1 \leq i \leq t)$ is irreducible and $\left(W_{i} / U_{i}\right) \gamma \neq 0$ for some $j=1$, $\ldots, s$; moreover, for each $j=1, \ldots, s$ and $i=0, \ldots,{ }_{t}$, we have that $U_{i} \cap$ $V_{\gamma_{j}}=W_{i+1} \cap V_{\gamma_{j}}$, and that the distinct members of the chain

$$
V_{\gamma_{j}}^{2}=\left(U_{0}\right)_{\gamma_{j}} \supset\left(U_{1}\right)_{\gamma_{j}} \supset \ldots\left(_{t}\right)_{\gamma_{j}}=0
$$

form a composition series of the $A^{\gamma}{ }^{j_{\text {-module }}} V_{\gamma_{j}}^{2}$.

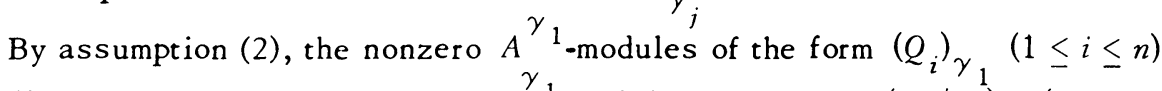
are all irreducible, and the nonzero $A^{\gamma}{ }^{1}$-modules of the form $\left(W_{i} / U_{i}^{1}\right)_{\gamma_{1}}(1 \leq$ $i \leq t$ ) are all irreducible. Since the $A^{\gamma_{1}}$-modules $V_{\gamma_{1}}^{1}$ and $V_{\gamma_{1}}^{2}$ have equivalent composition series, the two sets of irreducible $A^{\gamma}{ }_{1}$-modules are the same up to equivalence and order. Hence by assumption (3), the corresponding $B$-modules $Q_{i}$ are equivalent to the corresponding $B$-modules $W_{i} / U_{i}$. Continuing in this way for $\gamma_{2}, \ldots, \gamma_{s}$, and taking into account the equivalences established by the previous $\gamma_{j}$ 's, the choice of the $\gamma_{j}$ 's implies that $n=t$ and that the $B$ modules $Q_{i}(1 \leq i \leq n)$ are equivalent to the $B$-modules $W_{i} / U_{i}(1 \leq i \leq n)$, up to order. 
It remains to show that $U_{i}=W_{i+1}$ for all $i=0, \ldots, n$. But if this fails to hold, then for some $\gamma \in \Gamma$ and some $i=0, \ldots, n$, we have $\left(U_{i}\right)_{\gamma} \neq\left(W_{i+1}\right)_{\gamma}$. But this contradicts the fact that the $A^{\gamma}$-modules $V_{\gamma}^{1}$ and $V_{\gamma}^{2}$ have equivalent composition series, since the nonzero $A^{\gamma}$-modules of the form $\left(Q_{i}\right) \gamma$ are equivalent to the nonzero $A^{\gamma}$-modules of the form $\left(W_{i} / U_{i}\right)_{\gamma}$, up to order. Q.E.D.

Remark 11.9. The above proof of Theorem 11.1 shows that the last assertion of Theorem 11.1 remains valid if in place of the hypothesis that $V_{\gamma}^{1}$ and $V_{\gamma}^{2}$ have equivalent composition series as $A^{\gamma}$-modules for all $\gamma \in \Gamma$, we assume this only for $\gamma_{1}, \ldots, \gamma_{s}$ (defined in the proof of Theorem 11.1), and for all the other $\gamma \in \Gamma$ we assume that $V_{\gamma}^{1}$ and $V_{\gamma}^{2}$ are equivalent as $\mathbb{Q}_{\text {modules, }}$ and are finite sums of

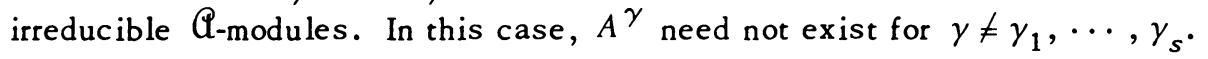

Corollary 11.10 to Theorem 11.1 (cf. Remark 9.4 and [ 15, Theorem 2.1 and Corollary 2.1]). Let $V^{1}$ and $V^{2}$ be B-modules such that $V^{i}=\Sigma_{\gamma \in \Gamma} V_{\gamma}^{i}(i=1,2)$, and suppose that $V^{1}$ is B-irreducible. Assume either that

(i) for every $\gamma \in \Gamma$, the $A^{\gamma}$-modules $V_{\gamma}^{1}$ and $V_{y}^{2}$ bave equivalent composition series (i.e., that they are equivalent), or that

(ii) for some $\gamma_{0} \in \Gamma$, the $A^{\gamma_{0}}{ }_{\text {-modules }} V_{\gamma_{0}}^{1}$ and $V_{\gamma_{0}}^{2}$ are nonzero and equivalent, and for every other $\gamma \in \Gamma, V_{\gamma}^{1}$ and $V_{\gamma}^{2}$ are equivalent as $\mathbb{G}$-modules and

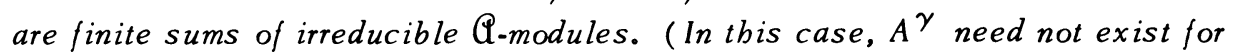
$\gamma \neq \gamma_{0}$.)

Then $V^{2}$ is B-irreducible and is equivalent to $V^{1}$.

Proof. The corollary follows immediately from Theorem 11.1 and Remark 1 1.9. Alternatively, the corollary follows directly and easily from Lemma 11.4 and assumptions (1), (2) and ( 3 ). Q.E.D.

Remark 11.11. If in the statement of Theorem 11.1, we assume in addition that $V^{2}$ has a composition series, then the last assertion of the theorem is a simple consequence of assumptions (1), (2) and (3).

\section{BIBLIOGRAPHY}

1. N. Bourbaki, Éléments de mathématique. XXVI. Groupes et algèbres de Lie. Chap. 1: Algèbres de Lie, Actualités Sci. Indust., no. 1285, Hermann, Paris, 1960. MR 24 \#A2641.

2. F. Bruhat, Sur les représentations induites des groupes de Lie, Bull. Soc. Math. France 84 (1956), 97-205. MR 18, 907.

3. J. Dixmier, a) Sur les représentations de certains groupes orthogonaux, C. R. Acad. Sci. Paris 250 (1960), 3263-3265. MR 22 \#5901.

b) Les $C^{*}$-algèbres et leurs représentations, 2ième éd., Cahiers Scientifiques, fasc. XXIX, Gauthier-Villars, Paris, 1969. MR 39 \#7442. 
c) Idéaux primitifs dans l'algèbre enveloppante d'une algèbre de Lie semi-simple complexe, C. R. Acad. Sci. Paris Sér. A-B 272 (1971), A1628-A 1630.

4. S. G. Gindikin and F. I. Karpelevič, Plancherel measure for Riemann symmetric spaces of nonpositive curvature, Dokl. Akad. Nauk SSSR 145 (1962), 252-255 = Soviet Math. Dokl. 3 (1962), 962-965. MR 27 \#240.

5. R. Godement, A theory of spherical functions. I, Trans. Amer. Math. Soc. 73 (1952), 496-556. MR 14, 620.

6. Harish-Chandra, a) On representations of Lie algebras, Ann. of Math. (2) 50 (1949), 900-915. MR 11, 77.

b) Representations of a semisimple Lie group on a Banach space. I, Trans. Amer. Math. Soc. 75 (1953), 185-243. MR 15, 100.

c) Representations of semisimple Lie groups. II, Trans. Amer. Math. Soc. 76 (1954), 26-65. MR 15, 398.

d) Representations of semisimple Lie groups. III, Trans. Amer. Math. Soc. 76 (1954), 234-253. MR 16, 11.

e) The Plancherel formula for complex semisimple Lie groups, Trans. Amer. Math. Soc. 76 (1954), 485-528. MR 16, 111.

f) The characters of semisimple Lie groups, Trans. Amer. Math. Soc. 83 (1956), 98163. MR 18, 318.

g) Spherical functions on a semisimple Lie group. I, Amer. J. Math. 80 (1958), 241-310. MR 20 \#925.

h) Some results on differential equations and their applications, Proc. Nat. Acad. Sci. U.S.A. 45 (1959), 1763-1764. MR 31 \#274.

i) Invariant eigendistributions on a semisimple Lie group, Trans. Amer. Math. Soc. 119 ( 1965), 457-508. MR 31 \#4862d.

7) S. Helgason, a) Differential geometry and symmetric spaces, Pure and Appl. Math., vol. 12, Academic Press, New York, 1962. MR 26 \#2986.

b) A duality for symmetric spaces with applications to group representations, Advances in Math. 5 (1970), 1-154. MR $41 \# 8587$.

8. A. W. Knapp and E. M. Stein, Interwining operators for semisimple groups, Ann. of Math.(2) 93 (1971), 489-578.

9. B. Kostant, On the existence and irreducibility of certain series of representations, Publication of 1971 Summer School in Math., edited by I.M. Gel'fand, Bolyai-Janós Math. Soc., Budapest (to appear).

10. R. A. Kunze and E. M. Stein, Uniformly bounded representations. III. Interwining operators for the principal series on semisimple groups, Amer. J. Math. 89 (1967), 385-442. MR $38 \# 269$.

11. J. Lepowsky and G. W. McCollum, $O_{n}$ the determination of irreducible modules by restriction to a subalgebra, Trans. Amer. Math. Soc. 176 (1973), 45-57.

12. K. R. Parthasarathy, R. Ranga Rao and V.S. Varadarajan, Representations of complex semi-simple Lie groups and Lie algebras, Ann. of Math. (2) 85 (1967), 383-429. MR $37 \# 1526$.

13. C. Rader, Spherical functions on semisimple Lie groups, Ann. Sci. École Norm. Sup. (to appear). 
14. G. Schiffmann, Intégrales d'entrelacement et fonctions de Whittaker, Bull. Soc. Math. France 99 (1971), 3-72.

15. N. Wallach, Cyclic vectors and irreducibility for principal series representations. II, Trans. Amer. Math. Soc. 164 (1972), 389-396.

16. D. P. Želobenko, Analogue of the Cartan-Weyl theory for infinite-dimensional representations of a semisimple complex Lie group, Dokl. Akad. Nauk SSSR 175 (1967), 24-27 = Soviet Math. Dokl. 8 (1967), 798-801. MR 35 \#5553.

DEPARTMENT OF MATHEMATICS, BRANDEIS UNIVERSITY, WALTHAM, MASSACHUSETTS 02154

Current address: Department of Mathematics, Yale University, New Haven, Connecticut 06520 